\title{
Resonant Raman, Hot, and Cold Luminescence of Iodine in Rare Gas Matrixes ${ }^{\dagger}$
}

\author{
J. Almy, K. Kizer, R. Zadoyan, and V. A. Apkarian* \\ Department of Chemistry, University of California, Irvine, California 92697-2025 \\ Received: September 13, 1999; In Final Form: December 7, 1999
}

\begin{abstract}
Spontaneous light emission (SLE) spectra induced by continuous wave excitation of iodine in rare gas matrixes is analyzed from the perspective of semiclassical molecular dynamics. The three-time correlation functions that describe the process are interpreted in terms of time circuits, where the reversibility of mechanics along specified paths establishes the distinction between resonant Raman (RR) and fluorescence. The observed hot luminescence (fluorescence from the vibrationally over-damped period of dynamics) is simulated, and the underlying dynamics analyzed to describe vibrational dephasing, formation of stationary states, and predissociation of $\mathrm{I}_{2}(\mathrm{~B})$ in matrixes. The extracted predissociation time of $3-5 \mathrm{ps}$ in matrixes agrees with time-domain measurements. The analysis allows the interpretation of liquid phase spectra which contain both structured and unstructured SLE as strictly RR, suggesting that the unstructured component is due to linear complexes. The spectra also contain vibrationally relaxed emissions from the cage-bound, doubly spin-orbit excited I*I* states, which are prepared via sequential two-photon excitation with an intermediate state lifetime of $2 \mathrm{~ms}$, assigned to $\mathrm{A}\left({ }^{3} \mathrm{P}_{1 \mathrm{u}}\right)$. The two main emission bands, which shift by $300 \mathrm{~cm}^{-1}$ in proceeding from $\mathrm{Ar}$ to $\mathrm{Kr}$ to $\mathrm{Xe}$, are assigned to $\mathrm{I}^{*} \mathrm{I}^{*}\left(1_{\mathrm{u}}\right) \rightarrow \mathrm{I}_{2}\left(\mathrm{a}\left(1_{\mathrm{g}}\right)\right)$, and $\mathrm{I}^{*} \mathrm{I}^{*}\left(0_{\mathrm{g}}{ }^{+}\right) \rightarrow \mathrm{I}_{2}\left(\mathrm{~B}^{\prime \prime}\left(1_{\mathrm{u}}\right)\right)$ transitions, and analyzed.
\end{abstract}

\section{Introduction}

As a prototype for condensed phase nonadiabatic dynamics, the $\mathrm{B}\left({ }^{3} \mathrm{P}_{0 \mathrm{u}}\right)$ state of molecular iodine has been the subject of extensive time domain studies in liquids, ${ }^{1-4}$ in clusters, ${ }^{5,6}$ under high pressure, ${ }^{7}$ and in rare gas matrixes..$^{8-10}$ Complementary to these measurements is the understanding of the frequency domain spectra of the molecule in condensed phases. The most extensive analysis in this context pertains to the resonant Raman (RR) spectra of iodine, which has been investigated in rare gas solids, ${ }^{11-13}$ in room-temperature liquids, ${ }^{14-16}$ and in cryogenic liquid Xe. ${ }^{17,18}$ Interest in these studies is motivated by the fact that the RR spectra relay information about ultrafast solvation dynamics on a time scale shorter than what can be achieved by direct time domain measurements, $t<50 \mathrm{fs}$, determined by electronic dephasing times. The dynamical information content of the RR spectra of iodine, in particular for the case of matrix isolation, has been scrutinized by a variety of theoretical approaches. ${ }^{19-22}$ The fluorescence of the molecule past this coherence time, but during over-damped vibrational relaxation where the residence time in a given vibrational state is shorter than the period of the state, $\tau_{\mathrm{r}}<2 \pi / \omega_{\mathrm{v}}$, is referred to as hot luminescence. ${ }^{23}$ This we contrast with cold luminescence, namely, emission during vibrational relaxation, whereby the system resides in stationary states for time scales longer than the characteristic period of nuclear motion. The information content of hot luminescence spectra is complementary to timedomain pump-probe measurements, in that both relay the classical nuclear dynamics of solvation and dissipation on chemically significant time scales, $\sim 0.1<t<10 \mathrm{ps}$, as we elaborate. Such an analysis also contains a description of the formation of stationary states. The cold luminescence spectra (the most common form of which is emission from vibrationally relaxed electronically excited states) in the case of matrix-

\footnotetext{
$\dagger$ Part of the special issue "Marilyn Jacox Festschrift".

* To whom correspondence should be addressed.
}

isolated iodine in the UV-vis spectral region belong to two categories: emission from cage-bound spin-excited states, ${ }^{24}$ and ion-pair states. ${ }^{25}$ An understanding of the band profiles associated with these spectra is quite informative, as they arise from configurations of complete electronic and nuclear solvation, far from the ground-state equilibrium structure. Characterization of the solvated potentials on various electronic surfaces of this prototype is useful.

In what follows, in Section II, we describe the experimental approach, and in Section III we present the experimental luminescence spectra of matrix-isolated iodine. In essence, we repeated the measurements of Andrews et al., ${ }^{11,12}$ who were interested mainly in the RR spectra. Our data are in excellent agreement with their measurements, even reproducing features that were originally assigned to impurities. Because the RR progressions observed in rare gas solids (RGS) have been scrutinized previously, after a succinct review of interpretations, we focus the discussion, Section IV, on the analysis of the hot and cold luminescence spectra. The interpretation of these spectra also allows significant conclusions with regard to spectroscopy and underlying interactions and dynamics in solid hosts versus liquid solvents, as we conclude in Section V.

\section{Experimental Section}

Initial attempts at preparing iodine in free-standing crystals of rare gases were mostly unsuccessful in isolating the molecule. Although RR spectra can be recorded with high sensitivity and good signal-to-noise is achieved in such crystals, see Figure 1, the spectra are dominated by dimers which are identified by their vibrational constant, $180 \mathrm{~cm}^{-1}$ as opposed to $220 \mathrm{~cm}^{-1}$ for the isolated molecule. ${ }^{11} \mathrm{We}$ thus resorted to preparation of thin film matrixes, as in the time domain studies. The matrixes were prepared by deposition of premixed gases. $\mathrm{I}_{2}$ was purified by trap-to-trap distillation, and its purity was verified by its vapor pressure. The room-temperature vapor above the solid was used to prepare the gas premix at an $\mathrm{I}_{2} / \mathrm{Rg}$ ratio of 1:3000. The films 


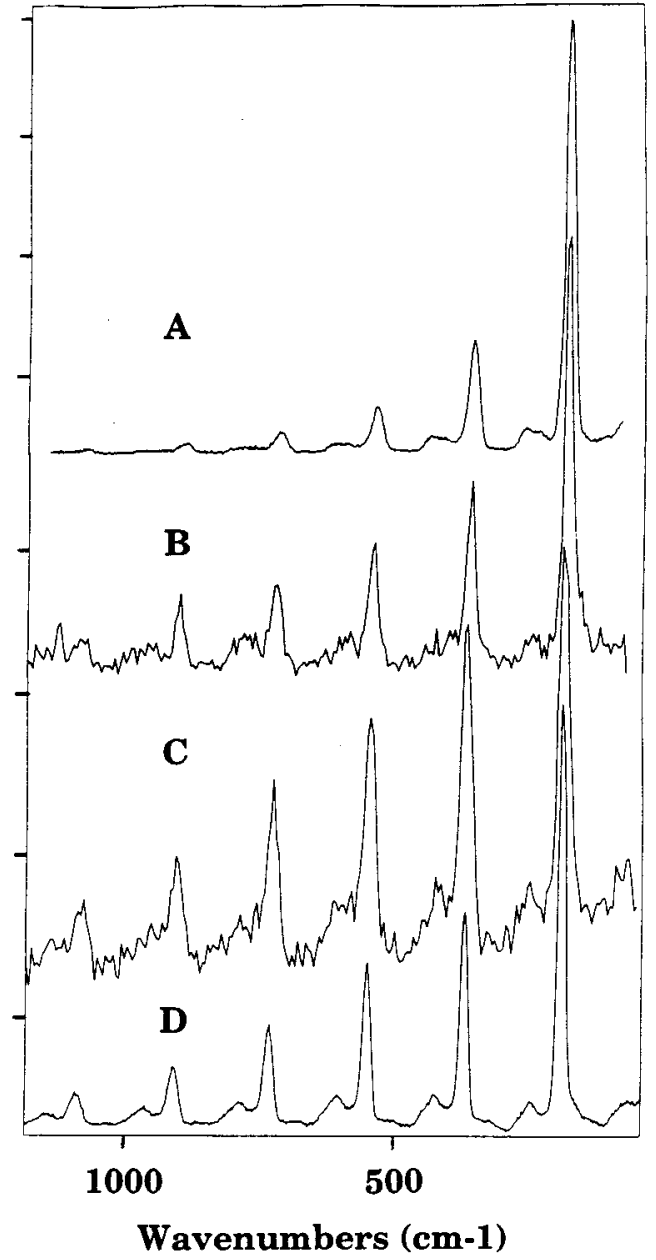

Figure 1. Resonant Raman spectra from a free-standing crystal of $\mathrm{Kr}$ doped with iodine, obtained at excitation wavelengths: (A) $457.9 \mathrm{~nm}$, (B) $488.0 \mathrm{~nm}$, (C) $496.5 \mathrm{~nm}$, and (D) $514.5 \mathrm{~nm}$. Even though the gas phase mole ratio of guest to host was 1:5000, the spectrum is entirely due to dimers, showing a vibrational progression with $\omega_{\mathrm{e}}=180 \mathrm{~cm}^{-1}$.

were prepared by spray-on deposition, onto a rhodium-coated copper substrate which was mounted on the tip of a closed cycle cryostat (base temperature of $11 \mathrm{~K}$ ). A variety of schemes were tried to optimize the isolation and to minimize optical scattering from the samples. The method that yielded reproducible results consisted of rapid deposition of the gas at a back pressure of 5-7 Torr, over the period of $\sim 5 \mathrm{~min}$. The same conditions were used for Ar, Kr, and Xe matrixes; in all cases the dimer signal was not detectable.

Three main lines of an Ar-ion laser were used as excitation source: $514.5,496$, and $488 \mathrm{~nm}$. Dispersive optics was used to further suppress plasma lines. The typical laser fluence at the sample was $1.6 \mathrm{~W} \mathrm{~cm}$. At higher laser intensities, rapid degradation of the sample through melting and sublimation occurs. Under the chosen conditions of operation, some laser annealing of the sample, particularly in the case of Ar, was evident in the first hour of irradiation. This could be verified by repeated scans over the period of $10-16 \mathrm{~h}$ required for recording a spectrum, and by noting that the scattering intensity in the first overtone (the largest RR peak) subsided. In highly scattering samples, due to photon diffusion, the effective excitation cross-section increases. As the sample anneals, this effect is reduced. The scattered light was dispersed through a 1-m double-grating monochromator, and detected using a thermoelectrically cooled photomultiplier in photon counting mode. Typical dark counts were $10-50 \mathrm{~Hz}$, whereas the largest
RR peaks had counts of $300 \mathrm{~Hz}$. This signal-to-noise ratio could only be achieved by sacrificing resolution, the line widths of the RR lines to be reported are instrument limited. Because relative intensities of $\mathrm{RR}$ peaks are of interest, we repeated scans, forward and back, to verify that intensities are stable during a particular scan. The monochromator and detection system were intensity calibrated using a $3000 \mathrm{~K}$ blackbody standard, and the calibration curve was used to correct the spectra. This correction is included in all of the reported relative intensities of observed overtones.

\section{Results}

In Figures 2-4, we provide raw spectra recorded at three excitation wavelengths, each in matrixes of $\mathrm{Ar}, \mathrm{Kr}$, and $\mathrm{Xe}$. The spectra are reported as a function of shift in wavenumbers from the excitation line, along with an absolute wavenumber scale. Stationary state emissions can be distinguished from Raman by the spectral shift of the feature with excitation wavelength. The latter occur at a fixed shift, whereas the former occur at a fixed wavelength independent of the excitation. In the spectra, the sharp RR progression of $I_{2}$ rides over a continuous background, which develops a maximum nearly independent of the excitation wavelength (most clearly seen for the $\mathrm{Kr}$ spectra), near $16200-17000 \mathrm{~cm}^{-1}$ in Ar through Xe. This continuous background, intermediate between the experimental definitions of Raman and stationary-state fluorescence, represents the hot luminescence upon which we amplify below.

Two structureless fluorescence bands occur in each of the spectra: at $15500 \pm 10 \mathrm{~cm}^{-1}$ and $14630 \pm 10 \mathrm{~cm}^{-1}$ in Ar, at $15290 \pm 10 \mathrm{~cm}^{-1}$ and $14485 \pm 10 \mathrm{~cm}^{-1}$ in $\mathrm{Kr}$, and at 14990 $\pm 10 \mathrm{~cm}^{-1}$ and $14300 \pm 20 \mathrm{~cm}^{-1}$ in Xe. Given the fact that these emissions occur at nearly twice the spin-orbit splitting of atomic iodine, $\Delta=7598 \mathrm{~cm}^{-1}$, it is not too difficult to guess their origin as $I^{*} I^{*} \rightarrow I_{2}$, i.e., transitions from the cage-bound states at the $\mathrm{I}\left({ }^{2} \mathrm{P}_{1 / 2}\right)+\mathrm{I}\left({ }^{2} \mathrm{P}_{1 / 2}\right)$ limit, terminating on states that correlate with $\mathrm{I}\left({ }^{2} \mathrm{P}_{3 / 2}\right)+\mathrm{I}\left({ }^{2} \mathrm{P}_{3 / 2}\right)$. Indeed, we previously reported and gave an analysis of emissions with the same upper state but terminating on the $\mathrm{I}\left({ }^{2} \mathrm{P}_{3 / 2}\right)+\mathrm{I}\left({ }^{2} \mathrm{P}_{1 / 2}\right)$ asymptote both in rare gas matrixes and in a variety of liquids. ${ }^{24}$ Note, however, that the I*I* asymptotes occur at $2 \Delta+D_{0}=27622 \mathrm{~cm}^{-1}$, which even with matrix stabilization cannot be directly reached with the $\mathrm{Ar}^{+}$-ion laser lines near $20000 \mathrm{~cm}^{-1}$. Using a variable speed chopper, we verify that these bands arise from two-photon sequential absorption of the continuous wave (CW) laser. In Figure 5, we show the emission intensity of the $14500 \mathrm{~cm}^{-1}$ band in $\mathrm{Kr}$ as a function of chopper frequency, using the 514 $\mathrm{nm}$ line for excitation. The data clearly show that the emitting state is prepared via sequential two-photon excitation, with an intermediate state lifetime of $2 \mathrm{~ms}$.

In addition to the unstructured emission bands, sharp fluorescence progressions that cannot be ascribed to molecular iodine are observed. One such progression is common to all matrixes, albeit with different relative intensities. This occurs in the spectral range between 14400 and $12400 \mathrm{~cm}^{-1}$ and can be fit to a final state with $\omega_{\mathrm{e}}=180 \mathrm{~cm}^{-1}$ and $\omega_{\mathrm{e}} x_{\mathrm{e}}=-5.9$. An additional sharp progression occurs in $\mathrm{Xe}$, in the spectral range between 13000 and $12000 \mathrm{~cm}^{-1}$. These emissions had also been observed by Andrews et al. who, on the basis of the observed negative anharmonicity assign it to a linear triatomic molecule. ${ }^{12}$ They suggested that a possible contaminant, namely IFeI, had formed on the stainless steel valves exposed to $I_{2}$. The assignment of these progressions remains uncertain, they could be the result of triatomic insertion products, such as IXeI, 


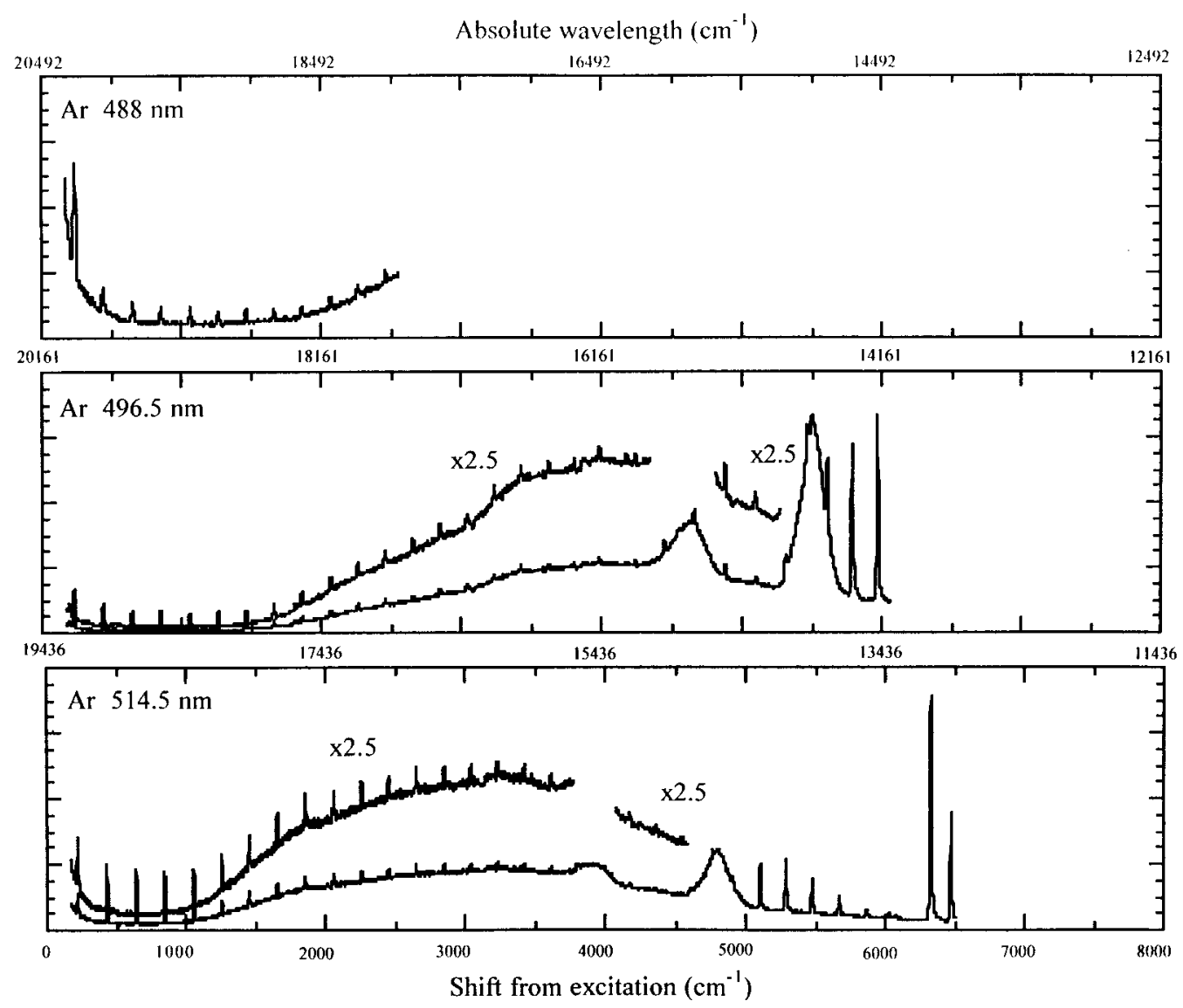

Figure 2. SLE spectra of matrix-isolated iodine in Ar.

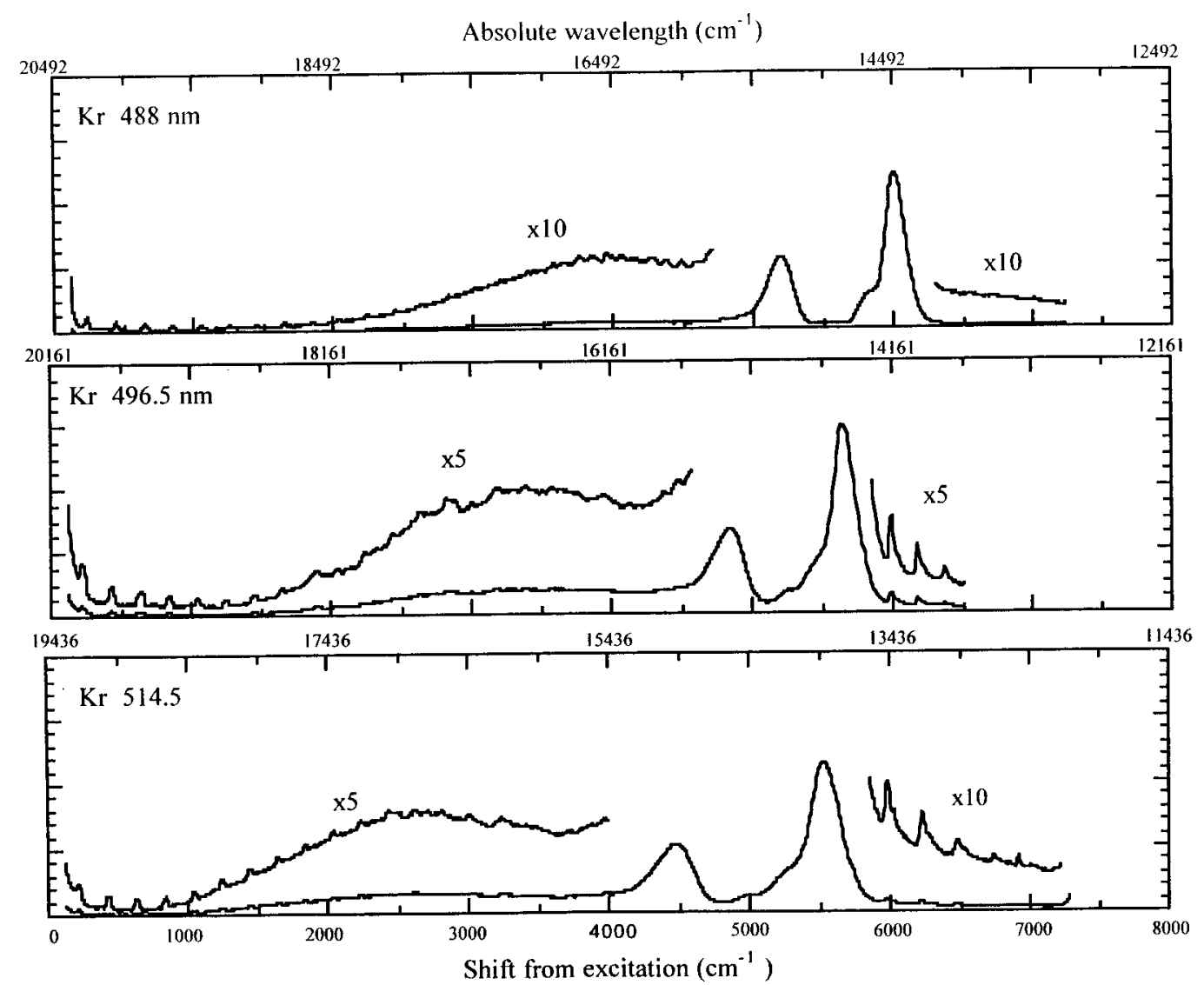

Figure 3. SLE spectra of matrix-isolated iodine in $\mathrm{Kr}$.

assuming Xe to be a common impurity in all rare gases used. Or, recognizing the fact that the progression shows a harmonic frequency the same as that of the RR progression of the dimer, it is likely that the emission is from intermolecular charge 
Absolute wavelength $\left(\mathrm{cm}^{-1}\right)$

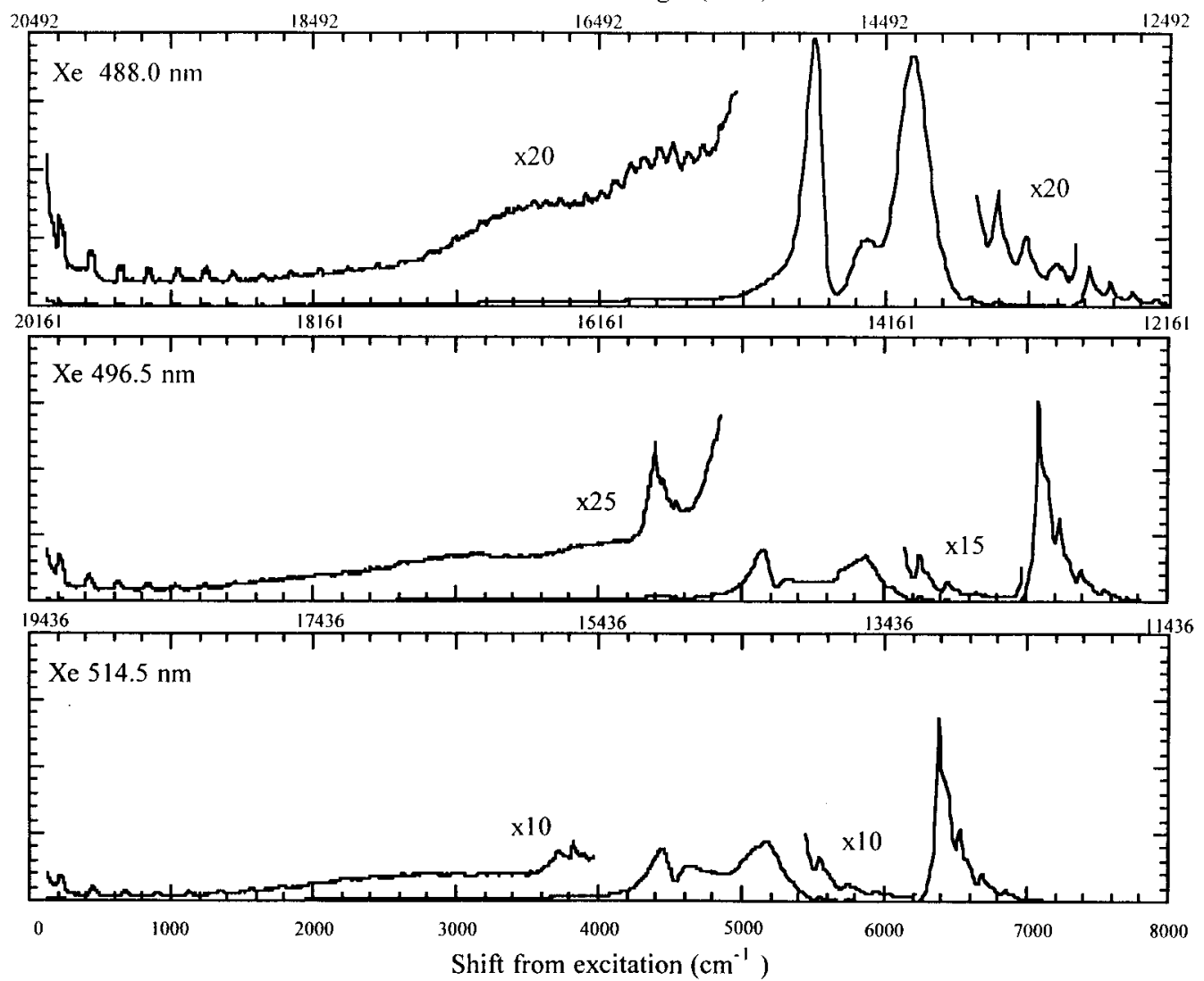

Figure 4. SLE spectra of matrix-isolated iodine in Ar.

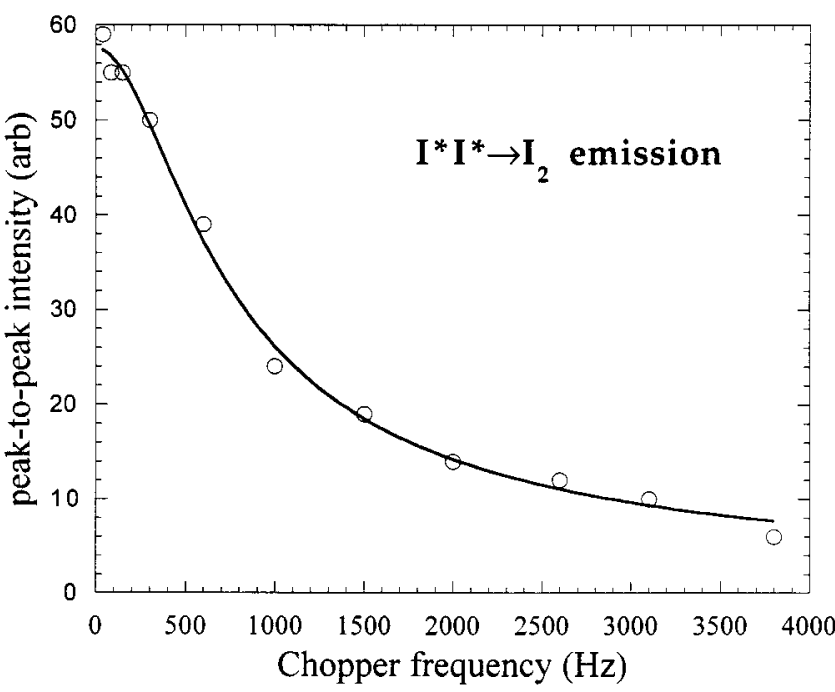

Figure 5. Peak-to-peak emission intensity of the $I^{*} I^{*}$ emission (at $15300 \mathrm{~cm}^{-1}$ ) in $\mathrm{Kr}$ as a function of chopper frequency. The data are fitted for linear response subject to sinusoidal drive, $\mathrm{I}(f)=\mathrm{I}(\mathrm{O}) /(1+$ $\left.(f \tau)^{2}\right)^{1 / 2}$, to extract $\tau=2 \mathrm{~ms}$.

transfer states. The necessary systematics have not been made for a firm conclusion.

The intensity profiles of the RR progressions are illustrated in Figure 6. The error bars are a reflection of the signal-tonoise ratio for the lower overtones, whereas for the higher overtones they reflect the difficulty in determining the baseline contribution from hot luminescence and interference from structured emission from stationary states (see expanded scale in the spectra of Figures 2-4). The data are corrected for intensity response of the system, and are obtained as areas of Gaussians fitted to individual RR lines over a carefully chosen sloping background. Several spectra were used for each sample and each wavelength, with the aims of reducing the uncertainty in this analysis. The break between $\mathrm{v}=12$ and $\mathrm{v}=17$ for the $514 \mathrm{~nm}$ data in $\mathrm{Kr}$ is due to blending of the RR lines with the irregularly structured fluorescence background in the same region. The modulations of the RR intensity profile should be interpreted with experimental error in mind. Note that the data are in good agreement with those of Andrews et al., although they fitted their data up to $\mathrm{v}=9$ to exponentials, and reported them as such. ${ }^{12}$ These smoothed curves, which have been used in prior theoretical analyses, ${ }^{19}$ are also shown in Figure 6.

\section{Discussion}

In dissipative systems, spontaneous light emission (SLE) can be decomposed into distinct contributions from coherent Raman emission and excited-state fluorescence. ${ }^{23}$ Although this distinction can be made in both frequency ${ }^{26-28}$ and time domain interpretations, ${ }^{29-31}$ the latter is the more natural for the analysis of condensed-phase systems. The sharp RR progression of some 25 overtones observed in the spectra of matrix-isolated iodine belong to the coherent process, and has been analyzed as such. ${ }^{17-22}$ Here, our main interest is the excited-state fluorescence component. Nevertheless, it is useful to establish the unity of the subject, to distinguish the information content of the various components of SLE, and to justify the approximations that are necessary in connecting spectroscopic observables to the underlying molecular dynamics of systems that involve more than $10^{2}-10^{3}$ degrees of freedom. In this context, we offer a perspective that has emerged from the semiclassical molecular dynamics formulation for spectral simulations, one that most intimately connects spectra to molecular mechanics of systems of large dimensionality. ${ }^{32}$ 


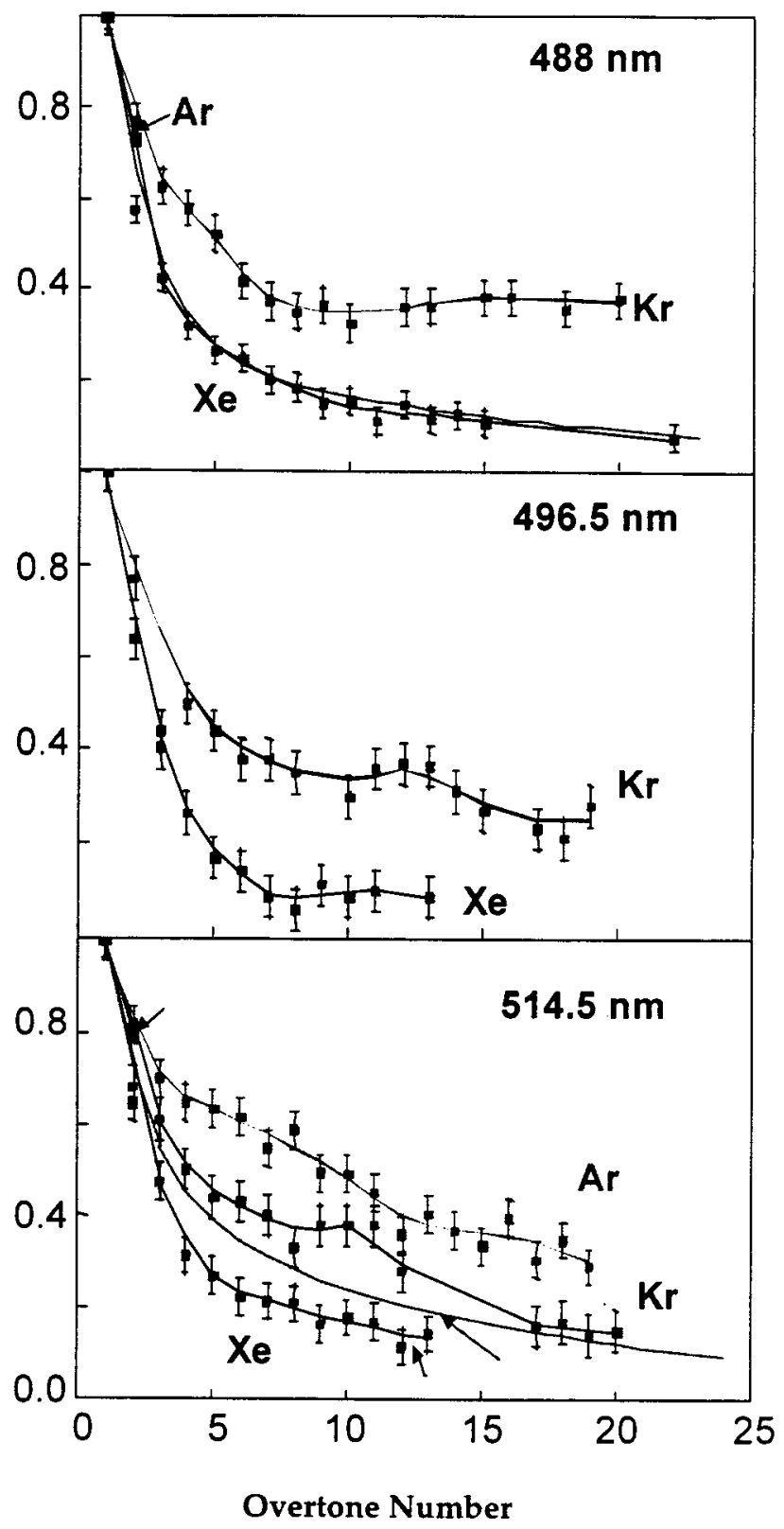

Figure 6. RR overtone intensities as a function of vibrational quantum number, $v$. The data are from intensity-calibrated spectra, and are normalized to the intensity of the first overtone in each case. The 488.0 and $514.5 \mathrm{~nm}$ excitation curves also show the curve fits obtained by Gyzybowski and Andrews.

Restricting ourselves to resonant transitions over the two electronic states of iodine, the $\mathrm{X}$ and $\mathrm{B}$ states, save for a proportionality constant, the SLE cross-section averaged over angles and polarizations can be expressed as: ${ }^{23,33}$

$$
\begin{aligned}
I_{\mathrm{SLE}}\left(\omega_{s}, \omega_{l}\right) & =\omega_{s}^{3} \omega_{l}\left|\int_{0}^{\infty} \mathrm{d} t \mathrm{e}^{i \omega_{s} t}\left\langle\varphi_{\mathrm{X}}(t) \hat{\mu}_{\mathrm{XB}} \varphi_{\mathrm{B}}(t)\right\rangle\right|^{2} \\
= & \omega_{s}^{3} \omega_{l} \mid \int_{0}^{\infty} \\
& \left.\mathrm{d} t \mathrm{e}^{i \omega_{s} t}\left\langle\varphi_{\mathrm{X}}(t) \hat{\mu}_{\mathrm{XB}} \mathrm{e}^{-i H_{\mathrm{B}} t / \mathrm{h}} \hat{\mu}_{\mathrm{BX}} \mathrm{e}^{-i \omega_{l} t} \varphi_{\mathrm{X}}(t)\right\rangle\right|^{2}
\end{aligned}
$$

Namely, the SLE radiated at $\omega=\omega_{s}$ is the result of the dipole projection of the wave function evolving on the excited B state onto the ground $\mathrm{X}$ state, with spectral distribution given by the square of the half-Fourier transform of the amplitude timecorrelation function contained in $\langle\ldots\rangle$. It is explicitly noted in eq 1 that the wave function on the B state evolves subject to the Born-Oppenheimer separated nuclear Hamiltonian, $H_{\mathrm{B}}$, and that this wave function was created by the CW field at $\omega_{1}$. SLE is a second-order process involving the two radiation fields of excitation and emission. Although clear in its statement, the implementation of eq 1 is impractical due to the large dimensionality of the system, and the fact that even at cryogenic temperatures we do not deal with pure states, but rather thermally populated statistical states of the extended system. The formulation of the correlation function in terms of densities, and their semiclassical propagation, is the more powerful framework for microscopic analyses of condensed-phase systems. By opening the square in eq 1 , instead of a time-correlation function in amplitudes, the three-time density correlation function is obtained. ${ }^{23} \mathrm{~A}$ three-time correlation results because the radiation-matter interaction, $\hat{\mu}_{i j}$, which appears twice in eq 1, will appear four times in the squared expression; and the possible time-orderings of the integral between four interactions are completely expressed in terms of the three time intervals among them. The formulation by Mukamel is particularly attractive in this regard, because it factors the correlation function into the field part and strictly material response functions, $R_{i}: 23$

$$
\begin{aligned}
& S_{\mathrm{SLE}}\left(\omega_{s}, \omega_{l}, t\right)=2 \operatorname{Re} \int_{0}^{\infty} \mathrm{d} t_{3} \int_{0}^{\infty} \mathrm{d} t_{2} \int_{0}^{\infty} \mathrm{d} t_{1} \\
& \left\{E_{l}\left(t-t_{1}-t_{2}-t_{3}\right) E_{l}^{*}\left(t-t_{2}-t_{3}\right) \mathrm{e}^{i \omega_{l} t_{1}+i \omega_{s} t_{3}} R_{1}\left(t_{3}, t_{2}, t_{1}\right)+\right. \\
& E_{l}^{*}\left(t-t_{1}-t_{2}-t_{3}\right) E_{l}\left(t-t_{2}-t_{3}\right) \mathrm{e}^{-i \omega_{l} t_{1}+i \omega_{s} t_{3}} R_{2}\left(t_{3}, t_{2}, t_{1}\right)+ \\
& \left.\quad E_{l}^{*}\left(t-t_{1}-t_{2}-t_{3}\right) E_{l}\left(t-t_{3}\right) \mathrm{e}^{-i \omega_{l} t_{1}-i\left(\omega_{l}-\omega_{s}\right)+i \omega_{s} t_{3}} R_{3}\left(t_{3}, t_{2}, t_{1}\right)\right\}
\end{aligned}
$$

with the three material response functions defined as

$$
\begin{aligned}
& R_{1}\left(t_{3}, t_{2}, t_{1}\right)= \\
& \left\langle\mathrm{e}^{i H_{\mathrm{X}} t_{1} / \hbar} \hat{\mu}_{X B} \mathrm{e}^{i H_{\mathrm{B}} t_{2} / \hbar} \hat{\mu}_{B X} \mathrm{e}^{i H_{\mathrm{X}} t_{3} / \hbar} \hat{\mu}_{X B} \mathrm{e}^{-i H_{\mathrm{B}}\left(t_{1}+t_{2}+t_{3}\right) / \hbar} \hat{\mu}_{B X} \rho_{x}\right\rangle \\
& R_{2}\left(t_{3}, t_{2}, t_{1}\right)= \\
& \left\langle\hat{\mu}_{X B} \mathrm{e}^{i H_{\mathrm{B}}\left(t_{1}+t_{2}\right) / \hbar} \hat{\mu}_{B X} \mathrm{e}^{i H_{X} t_{3} / \hbar} \hat{\mu}_{X B} \mathrm{e}^{-i H_{\mathrm{B}}\left(t_{2}+t_{3}\right) / \hbar} \hat{\mu}_{B X} \mathrm{e}^{-i H_{\mathrm{X}} t_{1} / \hbar} \rho_{x}\right\rangle \\
& R_{3}\left(t_{3}, t_{2}, t_{1}\right)= \\
& \left\langle\hat{\mu}_{X B} \mathrm{e}^{i H_{\mathrm{B}} t_{1} / \hbar} \hat{\mu}_{B X} \mathrm{e}^{i H_{\mathrm{X}}\left(t_{2}+t_{3}\right) / \hbar} \hat{\mu}_{X B} \mathrm{e}^{-i H_{\mathrm{B}} t_{3} / \hbar} \hat{\mu}_{B X} \mathrm{e}^{-i H_{\mathrm{X}}\left(t_{1}+t_{2}\right) / \hbar} \rho_{x}\right\rangle
\end{aligned}
$$

The content of eq 3 is readily visualized diagramatically, as in Figure 7. Although the double-sided Feynman diagrams are well-suited for this purpose, we find the complementary circuit diagrams given in the same figure to be a more transparent representation of the material response functions. To be clear about the notation, consider $R_{3}$, which constitutes the purely coherent Raman response. Reading eq $3 \mathrm{c}$ from right to left, the recipe is (a) start with the ground-state density, $\rho_{\mathrm{X}}$, and propagate it forward in time for the time interval $t_{1}+t_{2}$ subject to the $\mathrm{X}$ state Hamiltonian; (b) switch to the B potential, and propagate there for the interval $t_{3}$; (c) switch back to the $\mathrm{X}$ surface, reverse time, and propagate back for the time interval $t_{2}+t_{3}$; (d) switch to the B surface, and continue reverse time propagation for the interval $t_{1}$; (e) switch back to the X surface to close the circuit, to obtain a correlation point. In execution, the initial density can be sampled in a variety of representations, the coherent state representation $\int d q_{0} d p_{0}\left|q_{0}, p_{0}><q_{0}, p_{0}\right| \rho_{\mathrm{X}}$, being the more effective. ${ }^{32}$ The evolution of the density is then given by the swarm of weighted trajectories of minimum uncertainty Gaussians that carry phase. ${ }^{34}$ For a given trajectory, the correlation point corresponds to the overlap of the Gaussian with itself after closing the prescribed time-circuit. Implicit in the prescription is the resonance condition imposed by the field components 

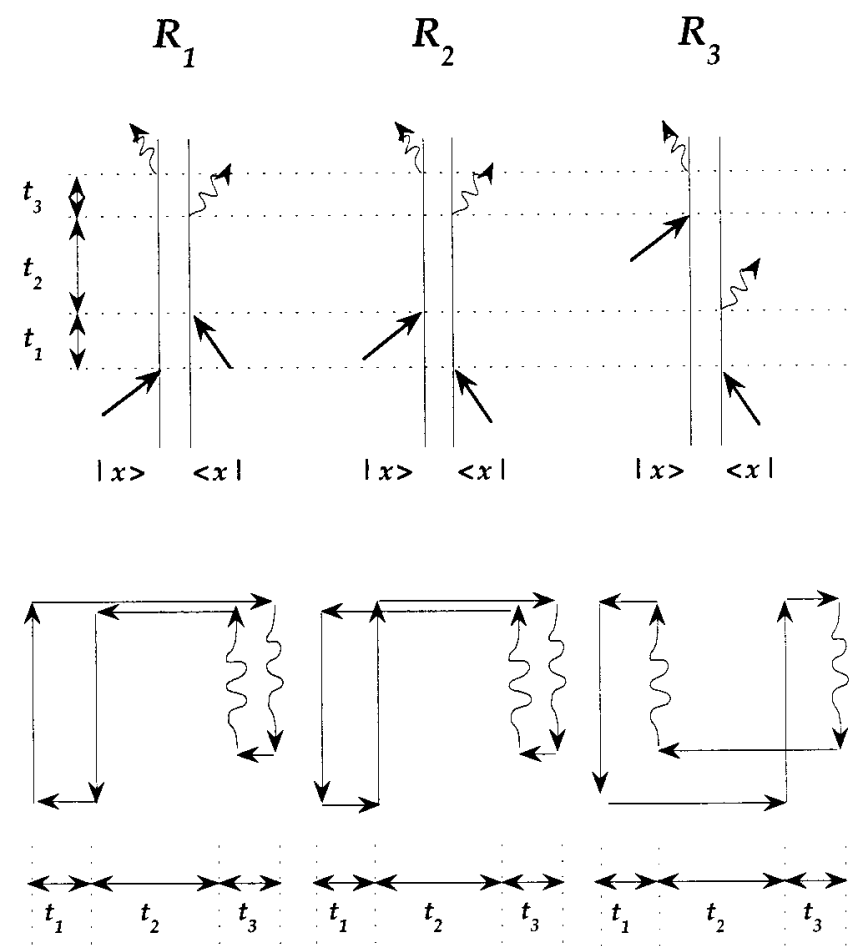

Figure 7. Double-sided Feynman diagrams and associated time-circuits for the three, three-time correlation functions that describe SLE.

which act as filters on their associated material response functions (eq 2), to ensure that the vertical jumps occur where the difference potential $\left|V_{\mathrm{B}}-V_{\mathrm{X}}\right|=\omega_{l}\left(\right.$ or $\left.\omega_{s}\right)$. Independent of the details of implementation, the correlation function evaluates the reversibility of the paths prescribed diagramatically in Figure 7. This interpretation of the prescribed circuits in terms of forward then backward time propagation has been recognized and implemented recently for spectral calculations. ${ }^{35}$

Classically, although trajectories obey strict time reversibility, the correlation function decays through energy redistribution. This occurs because the difference potential in systems of large dimensionality is multi-valued, therefore surface jumps along forward and reverse time propagation do not have to coincide in phase space. In smaller systems the correlation will revive after an initial decay due to redistribution, while in larger systems dissipation occurs when environmental noise precludes revivals. The quantum description allows for an additional mechanism of decay in the correlation function, namely electronic dephasing, or loss of quantum coherence, which can only occur over the loops in the circuit diagrams; or equivalently, when bra and ket states evolve on two different electronic states in the Feynman diagrams (when the system is said to be in a coherence). Otherwise, when the entire density is on one electronic surface, during the $t_{2}$ interval in each of the diagrams, the phase accumulated during forward time propagation is entirely reversed during reverse time propagation. Alternatively stated, because the density matrix can always be diagonalized by a suitably chosen basis set, $\rho(t)=\sum_{\alpha} e^{-i E_{\alpha} t / h}|\alpha\rangle p_{\alpha}\langle\alpha| e^{i E_{\alpha} t / \mathrm{h}}$ $=\sum_{\alpha}|\alpha\rangle p_{\alpha}\langle\alpha|$, where $p_{\alpha}$ is the probability of being in eigenstate $|\alpha\rangle$, then the relative phase between bra and ket states drops, and these sections of the circuits can be treated with a properly sampled swarm of classical trajectories (note that an overall phase between super-system and environment remains, ${ }^{36}$ which will lead to decoherence on a different time scale). The semiclassical treatment incorporates phases, through the classical action associated with each trajectory; moreover, it weights the contribution of each trajectory to the correlation through the sensitivity matrix, which measures the sensitivity of final positions and momenta to initial choices of the same. ${ }^{37,38}$ Thus, the sensitivity matrix has a built-in measure of the reversibility of paths. An exponential growth of sensitivity implying the access of chaotic regions of phase space, and therefore nonreversibility, or zero contribution to the correlation function. ${ }^{39}$ Finally, if we choose not to work in the nuclear eigenstate basis of the super-system, or when the coupling among such states is strong, then the redistribution of energy between modes produces vibrational dephasing, the mechanics of which can be easily highlighted by following the classical action associated with various coordinates, as we point out below.

The differences between the three contributions to SLE can be appreciated by considering the effect of collapsing the coherence loops. For $R_{1}$ and $R_{2}$ processes, when both $t_{1} \rightarrow 0$ and $t_{3} \rightarrow 0$, since bra and ket states always evolve simultaneously, we retrieve the classical description of absorption followed by time evolution of the population density on the excited state and subsequent emission. The changes of state are now described as classical Franck transitions with associated spectra determined by the reflection principle. It may also be appropriate to consider collapsing the $t_{3}$ loop but not the $t_{1}$ loop (or vice versa), i.e., to treat the absorption process through the single time-correlation function, as has already been done in the case of matrix-isolated $\mathrm{I}_{2} \cdot{ }^{21}$ The criterion that the coherence decay time be shorter than the characteristic period of nuclear motion determines the validity of such a collapse. In contrast, for $R_{3}$, as we collapse the coherence loop, i.e., as $t_{1} \rightarrow 0$ (or $t_{3}$ $\rightarrow 0$ ), then necessarily $\omega_{s} \rightarrow \omega_{i}$, and the entire diagram collapses to a nonevent. The $R_{3}$ diagram does not have a classical equivalent; it is strictly the coherent Raman contribution to SLE. In the absence of, or prior to the onset of dissipation and decoherence, all three response functions contribute to SLE as the continuous time-orderings of the same process, without a fundamental distinction between them.

A. The RR Progressions. The method of mixed-order semiclassical molecular dynamics in coordinate representation was introduced and applied to the analysis of $\mathrm{B} \leftarrow \mathrm{X}$ absorption, $\mathrm{A} \rightarrow \mathrm{X}$ emission, and the RR spectra of matrix-isolated $\mathrm{I}_{2}{ }^{21,22}$ Although the method makes approximations of convenience, it allows a microscopic interpretation of the spectra in terms of time correlation functions of the super-system, namely the chromophore and the bath. The simulations reproduced the nonmonotonic intensity profile of the RR overtones shown in Figure 6, the dynamical origin of which had been the subject of deliberation in prior works. ${ }^{17-19}$ The treatment specialized to the case of the $488 \mathrm{~nm}$ data in $\mathrm{Kr}$, which shows an intensity dip near $v=10$ followed by a recovery to a plateau..$^{22}$ A similar dip is observed in both $\mathrm{Kr}$ and $\mathrm{Xe}$, for excitation at $496.5 \mathrm{~nm}$ (see Figure 6). This intensity modulation could be understood in terms of interference between RR contributions from $\mathrm{B}\left({ }^{3} \pi_{\mathrm{u} 0}\right)$ and $B^{\prime \prime}\left({ }^{1} \Pi_{1 u}\right)$ surfaces, which are simultaneously accessed in this spectral region. Indeed, in their original experiments, Andrews et al. showed that the RR excitation profiles were bimodal, ${ }^{12}$ with peaks at the $\mathrm{B} \leftarrow \mathrm{X}$ and $\mathrm{B}^{\prime \prime} \leftarrow \mathrm{X}$ absorption maxima of 19700 and $18600 \mathrm{~cm}^{-1}$ as established through magnetic circular dichroism measurements. ${ }^{13}$ The same considerations apply to the liquid phase, and the presence of multiple scattering channels was recognized by Sension et al. in their analysis of excitation profiles in room-temperature liquids. ${ }^{14-16}$ The factors that shape the intensity profiles and their modulation include the relative absorption cross-sections of the $\mathrm{B} \leftarrow \mathrm{X}$ and $\mathrm{B}^{\prime \prime} \leftarrow \mathrm{X}$ transitions at a given excitation wavelength, the distance-dependent transition dipoles of the $\mathrm{B}$ 
$\rightarrow \mathrm{X}$ and $\mathrm{B}^{\prime \prime} \rightarrow \mathrm{X}$ transitions, differences in the solvated repulsive walls of the $\mathrm{B}$ and $\mathrm{B}^{\prime \prime}$ potentials, and the decay of the super-system correlation function on each of the surfaces with differences arising from the strength of coupling between chromophore and bath on each. Foregoing these details, the dynamical information content extracted in the past simulations are common to the full data set. Note that in the simulations the single-time amplitude correlation function (1) was used by approximate separation of the density into bath and $\mathrm{I}_{2}$ modes and the molecular eigenstates were used for projecting the evolving density. ${ }^{22}$ Here, we highlight the salient features of the dynamics that shape these spectra in terms of the reversibility of paths along the time-circuits of Figure 7.

First consider the time-circuits for the $R_{1}$ and $R_{2}$ response functions. Each contains the coherence loop over $t_{1}$, which is identical to the response function that determines the absorption spectrum. The reversibility of dynamics over this loop is limited to $30 \mathrm{fs}$, determined by the ballistic motion of the trajectories out of the Franck-Condon region along the I-I coordinate. Considered separately, the coherence loop over $t_{3}$ describes very similar dynamics, the main difference being that now the correlation function is for the vibrationally excited $\mathrm{X}$ state, therefore a similar time scale of decay of $\sim 30$ fs can be expected. Considered as a circuit, if we let $t_{2} \rightarrow 0$, the correlation function should be well-approximated as the product of those of the individual loops. In contrast, over the $t_{2}$ interval, the decay is due to energy redistribution which becomes nonreversible after the first hard collision in the bath at $t \sim 150$ fs. This decay has in the past been identified as the decay of the bath correlation function, $C_{\mathrm{B}}(t)$, to which we will have further recourse below. In sum, the time circuits associated with $R_{1}$ and $R_{2}$ response functions remain within $t<200 \mathrm{fs}$. We recognize that these contributions to the RR progression are not significant by noting that the line widths of the vibrational resonances are resolution limited to $\Delta \omega<10 \mathrm{~cm}^{-1}$ (in $\mathrm{Ar}$ ), and therefore determined by correlation functions that last for at least $3 \mathrm{ps}$. This can only be accomplished by the $R_{3}$ response function, with $t_{1}, t_{3} \sim 30 \mathrm{fs}$, and $t_{2} \sim 3 \mathrm{ps}$. Indeed, the main dynamics is now on the ground electronic surface, created after a short time propagation on the excited surface. On the excited state, the lattice is set into motion instantaneously, due to the difference in the electronic wave function of $\mathrm{I}_{2}$ in X and $\mathrm{B}$ states, and therefore the difference in forces along the chromophore-bath coordinate. The B state propagation must be short enough to ensure reversibility of the bath dynamics; nevertheless, given the very steep repulsive potential reached, even a short propagation ensures that both bath and chromophore are created with vibrational excitation in the $\mathrm{X}$ state. Along the $\mathrm{I}-\mathrm{I}$ coordinate, $\mathrm{v}=10$ is prepared at $t \sim 15 \mathrm{fs}$, whereas $\mathrm{v}=25$ is prepared at $t \sim 30 \mathrm{fs} .{ }^{22}$ The decay of intensity as a function of overtone number then measures the decay of $R_{3}$ along the $t_{1}$ and $t_{3}$ time intervals, whereas the spectral line widths determine the decay of $R_{3}$ along the $t_{2}$ interval, i.e., the vibrational redistribution on the ground electronic surface. Whether this decay involves relaxation of the vibrational states of $\mathrm{I}_{2}(\mathrm{X})$, or simply vibrational dephasing of this mode which is not an eigenstate of the system, cannot be established on the basis of line widths alone.

Emission past $\sim 100$ fs of time evolution on the B state, and for the duration prior to complete vibrational cooling, should be interpreted using the $R_{1}$ and $R_{2}$ diagrams with collapsed coherence loops, i.e., in terms of classical absorption and reemission of a population. This is the treatment used for the interpretation of the hot luminescence.

B. Hot Luminescence. The RR progressions in the solid matrixes ride over a continuous background that tails off toward the excitation energy, and that develops a broad maximum nearly independent of the excitation wavelength, near 17000 $\mathrm{cm}^{-1}$. Although this diffuse band must be assigned to fluorescence from the B state, because the electronic origin of the B state occurs at $T_{\mathrm{e}} \sim 15000 \mathrm{~cm}^{-1}$, it is clear that this does not correspond to vibrationally relaxed emission (which would be expected to produce a long progression in the near-IR, near $11000 \mathrm{~cm}^{-1}$ ). Indeed, predissociation should preclude the B state population from reaching the bottom of the potential. It is possible to estimate the time scale over which fluorescence occurs. Thus, from the relative integrated areas of the sharp RR progression to the diffuse fluorescence, we can estimate the ratio of time scales for the two components of spontaneous light emission as

$$
\tau_{\mathrm{fl}} / \tau_{\mathrm{RR}}=\int I_{\mathrm{fl}}(\omega) \omega^{-3} \mathrm{~d} \omega / \int I_{\mathrm{RR}}(\omega) \omega^{-3} \mathrm{~d} \omega \sim 10^{2}
$$

Because RR is associated with coherences that only spend $\tau_{\mathrm{RR}}$ $\sim 30 \mathrm{fs}$ on the B state, then $\tau_{\mathrm{fl}} \sim 3 \mathrm{ps}$ is the time scale of fluorescence from the population on the B state, well past the decay of coherences. This estimate is in good agreement with real time pump probe measurements of the evolving density on the B state, in which the transients are observed to decay in 4.5 ps, determined by predissociation rates. ${ }^{8}$ This also clarifies that although the estimate is two orders of magnitude more intense than the RR progression, because the radiative lifetime of the $\mathrm{B}$ state is $10^{-6} \mathrm{~s}$, we are monitoring a process of rather low quantum yield of $10^{-3}$. In the absence of predissociation, this hot luminescence would constitute a negligibly small tail of the vibrationally relaxed emission. Thus, it is the predissociative nature of the $\mathrm{B}$ potential that allows observation of the hot luminescence, which constitutes the frequency domain observable associated with dynamics that evolves on picosecond time scales as observed in time-resolved pump-probe measurements.

The picosecond time scale dynamics of $\mathrm{I}_{2}(\mathrm{~B})$ isolated in matrixes, and in particular in solid $\mathrm{Kr}$, was analyzed through classical molecular dynamics simulations which were inverted to reproduce the pump-probe observables. ${ }^{8,9}$ The same type of simulations are used here, but now we use the classical trajectories to reproduce the hot-luminescence spectrum. We examine the case of solid Xe, using a 512 atom simulation box with molecular iodine introduced in a divacancy of an otherwise perfect fcc lattice. The simulation cell is first thermalized at 40 $\mathrm{K}$, using a ground state molecular potential with minimum shifted to lie vertically below the B state potential for excitation at $514 \mathrm{~nm}$. The potential is then suddenly switched, and the Newtonian equations of motion are propagated using atomatom pair potentials. The Morse form is used for the I-I interaction, and $\mathrm{I}-\mathrm{Xe}$ interactions are treated as $\mathrm{Xe}-\mathrm{Xe}$ Lennard-Jones potentials. The latter approximation has proved satisfactory in the past. ${ }^{4}$

An understanding of the pattern of energy flow from molecule to lattice, and its spectral signature are the aims of the present analysis. A useful means of quantifying this is to consider the energy loss by the molecule as a function of its classical vibrational period, which is tracked by counting recursions of the I-I coordinate to the steep inner turning point, $R_{l}$. In Figure 8 we provide the period dependence of the ensemble-averaged difference in potential energy at the inner turning point:

$$
\left\langle\Delta V_{\mathrm{BX}}\left(R_{l}\right)\right\rangle=\frac{1}{N} \sum_{i=1}^{N}\left[V_{\mathrm{B}}\left(R_{l}\right)-V_{\mathrm{X}}\left(R_{l}\right)\right]
$$




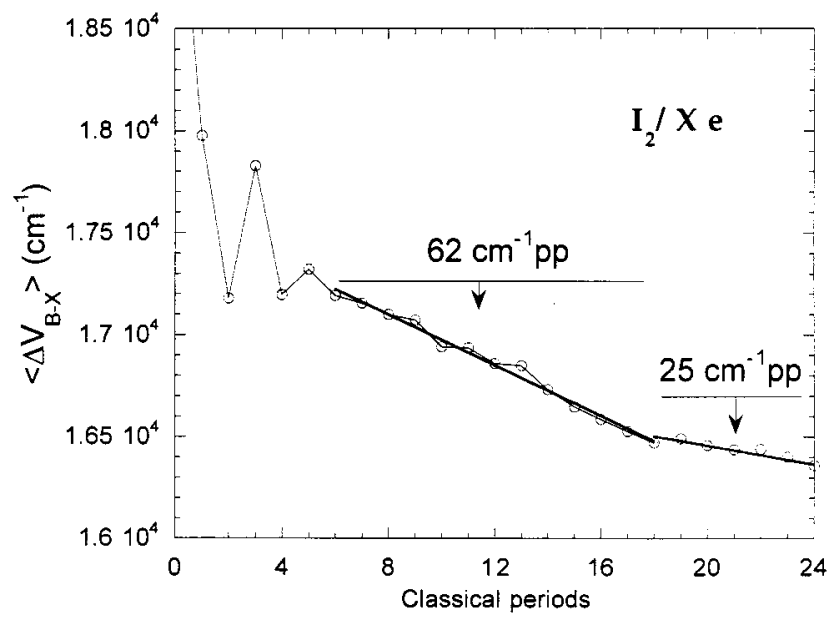

Figure 8. Energy loss as a function of classical periods. The points represent the average of 600 trajectories. The excitation energy at $t=$ 0 is $19455 \mathrm{~cm}^{-1}$ meant to simulate the $514 \mathrm{~nm}$ excitation.

where $N=600$ is the number of trajectories used. Because the inner turning point remains in the Franck-Condon window for emission, the plot provides a direct spectral map of the timedependent population. The observed energy flow pattern is typical for excitation in the anharmonic part of the B state potential, near its dissociation limit, as is the case for all of the RR excitation wavelengths. In the first period of motion, consisting of the first large-amplitude collision with the lattice, some $1500 \mathrm{~cm}^{-1}$ of energy is lost. A highly nonlinear phase of dynamics, with bidirectional energy flow, lasts for $3-5$ periods. This is followed by a linear decay at a rate of $60 \mathrm{~cm}^{-1}$ per period. A turnover in the linear decay rate occurs near the 15 th period, after which the energy decays at a rate of $25 \mathrm{~cm}^{-1}$ per period. The turnover can be correlated with structural relaxation of the cage.

These decay rates can be put in proper perspective given a knowledge of the natural period of motion in the solvated potential, namely, knowledge of the spacings among the quantized vibrations. Vibrational numbers may be assigned on the basis of the quantization of action:

$$
\oint S(t) \mathrm{d} t=\oint\left[T_{\mathrm{I}_{2}}(t)-V_{\mathrm{I}_{2}}(t)\right]=n \hbar
$$

where the integral is over the classical period of motion. In practice, it is convenient to propagate trajectories that carry phase, $\delta[r-r(t)] \exp (i S(t) / \hbar)$, and to count zero crossings of the real part $\cos (S(t) / \hbar)$. A representative example is shown in Figure 8 , along with counts of zero-crossings from inner turning point to inner turning point (see inset). The inset in Figure 9 also illustrates the essence of vibrational dephasing, in the form of phase variations at turning points. According to the numbering, the molecule is prepared in $\mathrm{v}=40$, and jumps down to $\mathrm{v}=25$ in one classical period. During the strongly nonlinear dynamics of the first four periods, the molecule may gain or lose 2-4 quanta per period in hard collisions with the immediate cage, with the main impact concentrated on atoms around the molecular belt. As the cage atom vibrations are damped out, the molecule first lingers near $\mathrm{v}=23$ in a distorted cage, then relaxes down to $\mathrm{v}=9$ in 15 classical periods. This corresponds to the initial linear energy flow regime of Figure 8, but now we establish that a loss of $60 \mathrm{~cm}^{-1}$ per period corresponds to spending, on average, one period per level. The turnover in the rate of energy flow, which accompanies structural relaxation of the cage, occurs at $v=7$, where now the molecule starts to spend several periods in each vibrational level. According to
Figure 8 , the turnover occurs at a difference potential energy of $16500 \mathrm{~cm}^{-1}$, a region wherein the experimental spectra of Figure 4 the fluorescence background develops structure in the form of sequences with $\sim 100 \mathrm{~cm}^{-1}$ spacing. The simulated vibrational spacing at $v=6$ is $110 \mathrm{~cm}^{-1}$.

To simulate the emission spectrum from the strongly damped vibrational population, it is necessary to incorporate population leakage due to predissociation. Moreover, because large amplitude motions are involved, it is necessary to account for the distance-dependent transition dipole, $\mu_{\mathrm{BX}}(R)$. For the former, we have implemented explicit Landau-Zener curve-crossing algorithms, with variable coupling strengths and curve-crossing locations. As determined previously from the analysis of timedomain data, a consistent treatment is only possible by placing the crossing near the bottom of the B potential. ${ }^{4}$ Because this is far removed from the evolving population, an exponential decay of the population yields results indistinguishable from the explicit treatment. With regard to the transition dipole, there is significant disagreement in the literature regarding its functional form. In Figure 10 we show the functions determined by Tellinghuisen ${ }^{40}$ and Lamrini et al., ${ }^{41}$ both of which are based on the analysis of experimental data. We have used both forms in the simulations. We carry out the spectral inversion of the trajectory data by first dressing the bare trajectories with Gaussians of width equal to the zero-point vibration on the B state, then reflect the smoothed distance distribution over the $\mathrm{B}-\mathrm{X}$ potential:

$$
\begin{aligned}
& I(\omega)=\sum_{i} \int \mathrm{d} t\left(1-C_{\mathrm{b}}(t)\right) \mathrm{e}^{-t / \tau} \int \times \\
& \mathrm{d} r e^{-\delta\left(r-R_{i}\right)^{2}}\left[\Delta V\left(R_{i}(t)\right)\right]^{3}\left|\mu_{\mathrm{BX}}\left(R_{i}(t)\right)\right|^{2} \delta\left[\Delta V\left(R_{i}(t)\right)-\hbar \omega\right]
\end{aligned}
$$

in which the summation is over the members of the trajectory ensemble. The first term in parentheses ensures that the RR emission is not included in the hot luminescence, using the previously determined bath correlation function $C_{\mathrm{b}}(t)$ which may be approximated as ${ }^{21}$

$$
\begin{aligned}
C_{\mathrm{b}}(t) & =1 & & t<50 \mathrm{fs} \\
& =\exp \left[-\frac{(t-50)^{2}}{80}\right] & & t>50 \mathrm{fs}
\end{aligned}
$$

The exponential decay in (eq 7) accounts for predissociation, and the delta function accomplishes the reflection of the spatial distribution from the difference potential. With a choice of a predissociation constant $\tau=4.5 \mathrm{ps}$, the same as that previously determined through time-domain measurements in solid $\mathrm{Kr}$, the simulated signal reproduces the expected features of the hot luminescence in $\mathrm{Xe}$, as shown in Figure 11. With the same procedure as outlined for $\mathrm{Xe}$, trajectory data previously used for simulations of pump-probe signals in $\mathrm{Kr}$ were inverted to reproduce the hot luminescence spectrum in Figure 12. The features of note, and their sensitivity to the various parameters are as follows:

(a) Consistent with the experiment, the hot luminescence starts after a spectral shift of $1000 \mathrm{~cm}^{-1}$. This cannot be reproduced without filtering out the coherent emission during the initial ballistic evolution out of the Franck-Condon region. Because $C_{\mathrm{b}}(t)$ decays on the time-scale of $150 \mathrm{fs}$, the blue-most contribution to emission occurs upon bond compression, after the first collision of the molecule with the cage, after the loss of $\sim 1500 \mathrm{~cm}^{-1}$ to the lattice (see Figure 8 ).

(b) As in the experiment, the simulated luminescence peaks at a spectral shift of $3000 \mathrm{~cm}^{-1}$, which according to Figure 8 


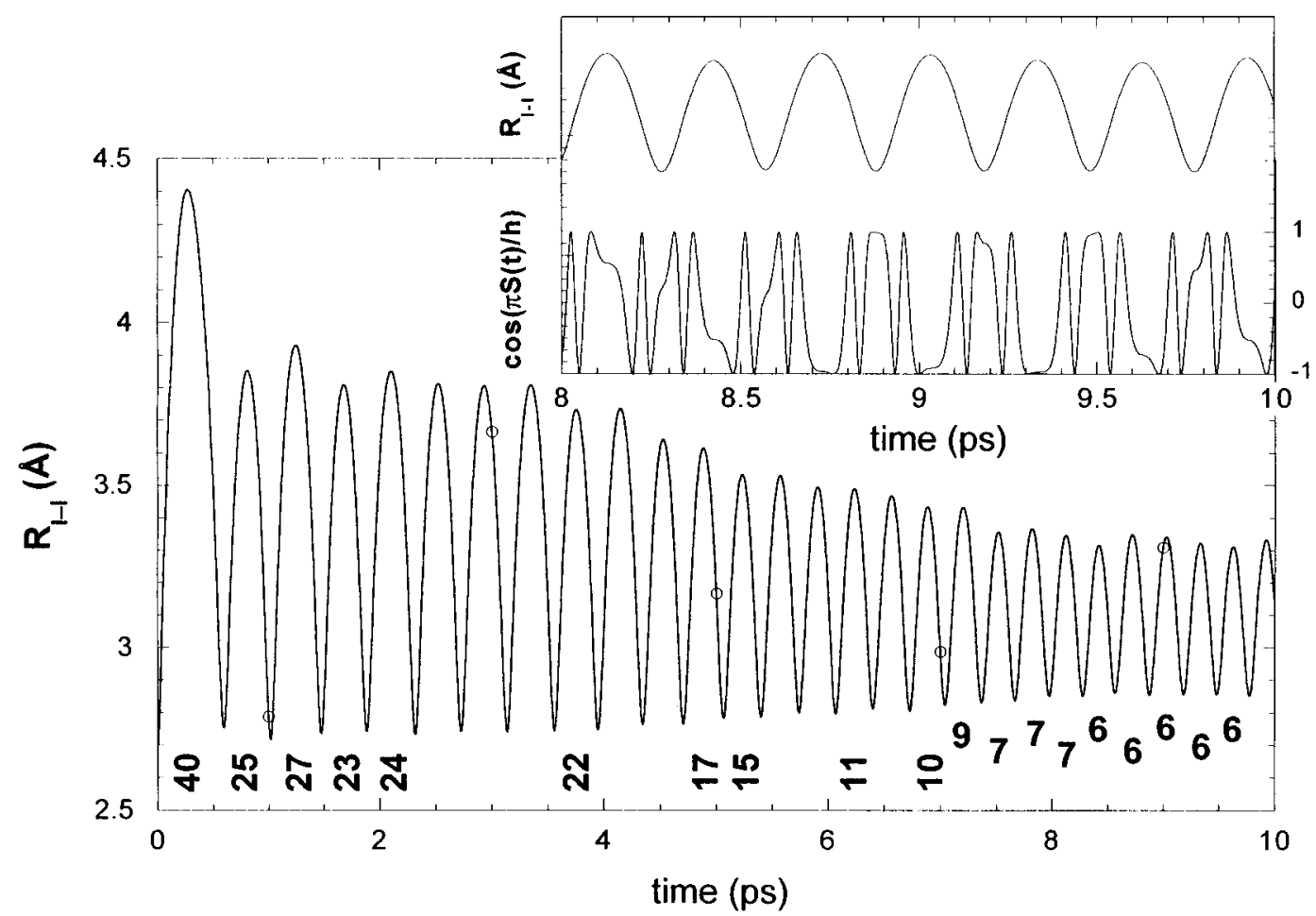

Figure 9. Vibrational assignment for a representative trajectory. The $I-I$ internuclear distances are shown as a function of time, and the quantization of action, $S(t)=\oint p d q$, along the I-I coordinate is used to assign vibrational quantum numbers for each classical period between inner turning points. In the inset, a plot of $\cos (S(t) / 2 h)$ is shown, where the number of zero crossings directly yields vibrational quantum number $v$. Where $v$ remains the same for repeated classical periods, vibrational dephasing appears as the nonstationary values of the action at turning points. In the over-damped region, where $v$ changes by several number between classical periods, the assignment is not meaningful; a strictly classical description of the dynamics is the more appropriate.

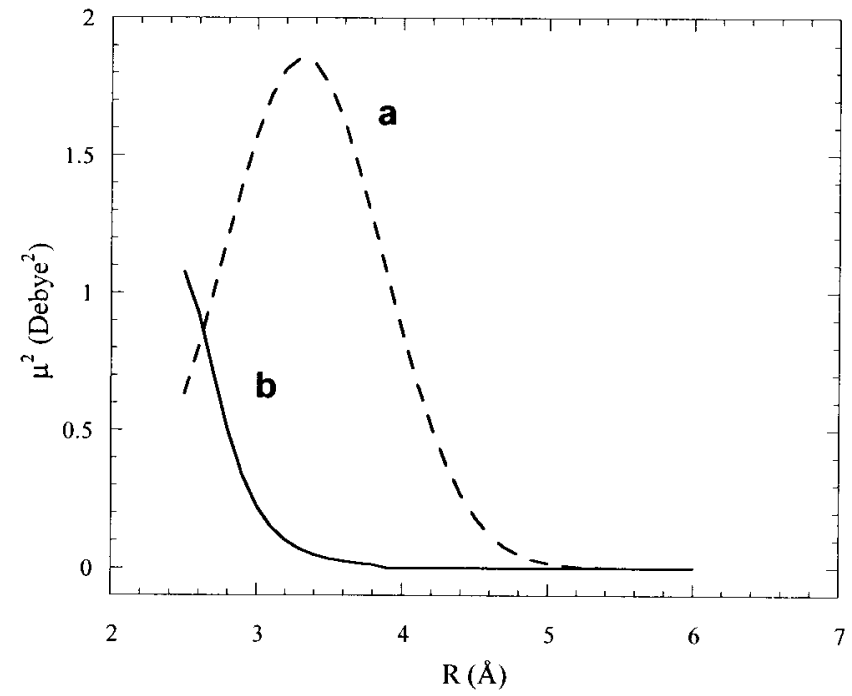

Figure 10. The squared transition dipole, $\left|\mu_{\mathrm{BX}}\right|^{2}$, as a function of internuclear distance is shown: (a) from ref 40 , (b) from ref 41.

corresponds to emission from the turnover region. The location of the maximum is sensitive to both the predissociation rate and the choice of the functional form of the transition dipole, as would be expected from the comparison in Figure 10. Tellinghuisen's functional form, however, also leads to spectral density throughout the recorded region, which cannot be compensated by any choice of predissociation rates. However, the monotonically decaying function of Lamrini et al. leads to a decay of spectral density, consistent with the experiment in the windows between fluorescence features.

(c) The simulation reproduces the spectral width, full width at half-maximum $(\mathrm{fwhm})=2000 \mathrm{~cm}^{-1}$, which is controlled by

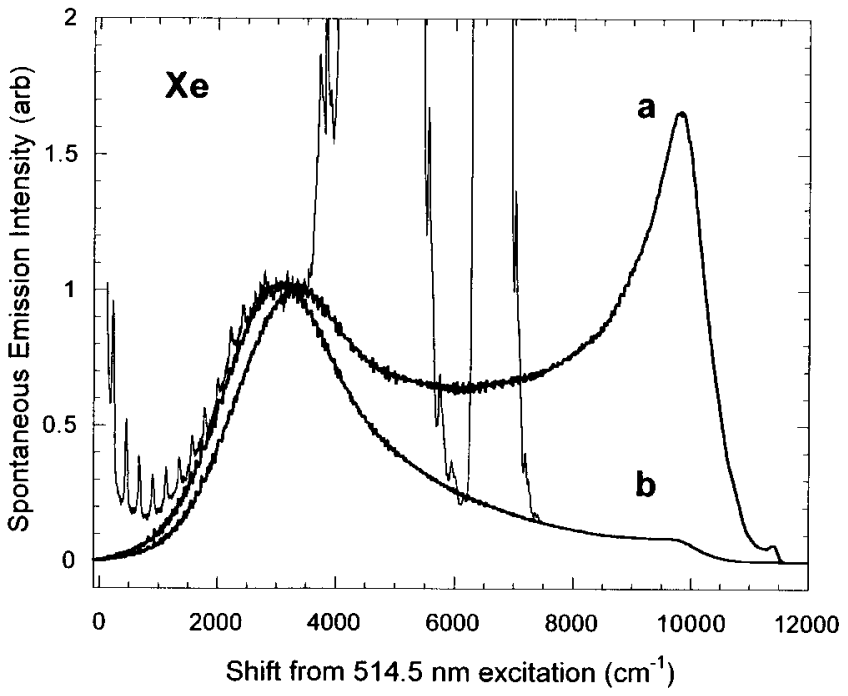

Figure 11. Comparison of simulated hot-luminescence spectrum with experiment in Xe using the transition dipole functions of (a) Tellinghuisen, ${ }^{40}$ and (b) Lamrini et al. ${ }^{42}$

the combination of predissociation, the distance dependence of the transition dipole, and vibrational energy flow dynamics. A close comparison of the hot luminescence bands in $\mathrm{Ar}, \mathrm{Kr}$, and $\mathrm{Xe}$ indicates that the initial increase of the luminescence becomes successively delayed with small shifts in the location of the maximum. This is captured in the simulations of $\mathrm{Kr}$ and $\mathrm{Xe}$, and because the same predissociation rates were used, would imply that the vibrational energy loss upon the first collision with the cage determines the shift. By varying the assumed predissociation rate, acceptable fits are obtained for $\tau=4.5 \pm$ $1.5 \mathrm{ps}$. The dependence of the predissociation rate on the matrix, 


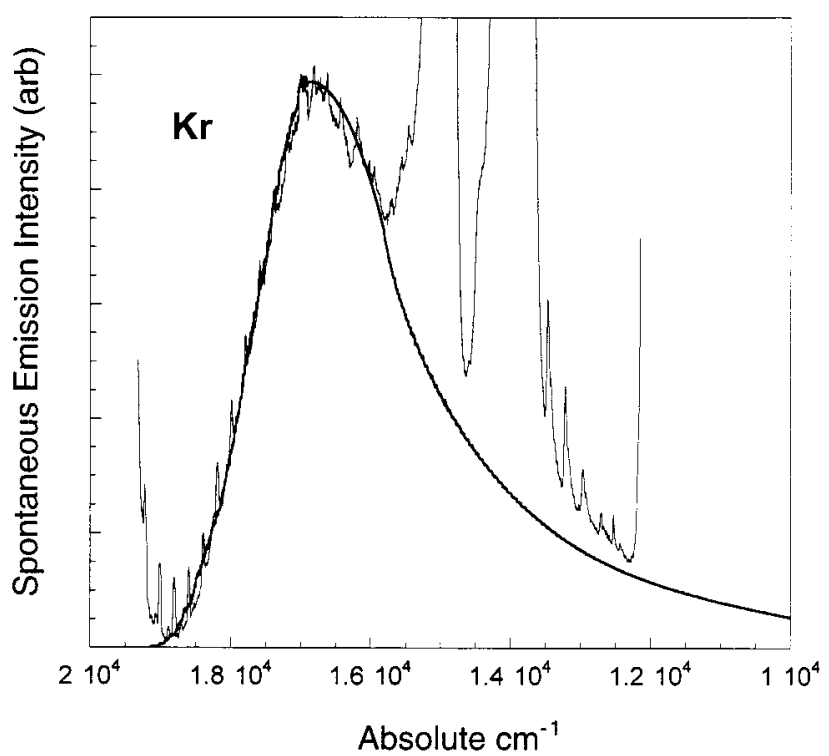

Figure 12. Comparison of simulated hot-luminescence spectrum with experiment in $\mathrm{Xe}$.

Ar through Xe, appears to be within this uncertainty of $30 \%$. In short, consistent with the time-resolved measurements, the hot-luminescence analysis clearly indicates that the predissociation probabilities are of order $10^{-2}$ per classical period, nearly 2 orders of magnitude smaller than the prediction by the most recent theoretical simulations. ${ }^{42}$

The simulations fail to reproduce the structured emission, which although most prominent in the $488 \mathrm{~nm}$ excitation spectra in Xe, also occurs in $\mathrm{Kr}$. This consists of overlapping sequences with a spacing of $100 \mathrm{~cm}^{-1}$. Both the emission wavelength and spacing in the structured emission is consistent with the nascence of vibrational eigenstates near $\mathrm{v}=7$. Moreover, the breadth of these features is consistent with the limitation of the residence time of the population in these states to $2-3$ periods. To reproduce this structure, at least a semiclassical simulation of the $R_{1}$ and $R_{2}$ response functions is necessary (circuits with collapsed $t_{1}$ loop, but not $t_{3}$ loop). Nevertheless, it is remarkable that the classical trajectories qualitatively predict the effect.

C. RR in the Liquid Phase. In view of the above analysis, the liquid-state RR spectra of iodine reported by Xu et al. acquire a unique interpretation. ${ }^{17,18}$ The spectra show a clear RR progression of some 15 overtones, riding over a continuum which is distinctly different from the hot luminescence band observed in matrixes. The continuum, which contains an integrated intensity three times that of the discrete lines, occurs in the same spectral range as the progression: it starts at zeroshift, peaks at a Stokes shift of $1500 \mathrm{~cm}^{-1}$, and decays as the intensity in the discrete progression decays. Contrary to the interpretation offered earlier, this continuum cannot be attributed to hot luminescence (or scattering-induced fluorescence). ${ }^{17}$ The observed continuum profile in the liquid phase can be reproduced using (eq 7), however, only under the assumptions that (a) the emission is coherent, by setting $\left(1-C_{\mathrm{b}}(t)\right)=1$; (b) if we assume that the coherence decays within $27 \mathrm{fs}$ through the exponential decay function provided in (eq 7). More simply put, because the continuum emission starts at zero-shift and occurs in the same spectral region as the RR progression, it occurs during the first $20 \mathrm{fs}$ of ballistic motion of the wave packet on the B state, namely, within the period of electronic coherence. The continuum must also be identified as RR.

The origin of two distinct RR spectra in the liquid phase, one discrete and one continuous, can be traced to the solvation structure. Using anisotropic pair potentials, we have previously shown that in liquid $\mathrm{CCl}_{4}$ the first solvent shell around groundstate iodine is structured, with a 3:1 ratio of linear versus T-geometries. ${ }^{4}$ The same also occurs in the $\mathrm{I}_{2}-\mathrm{Rg}$ binary complexes in molecular beams. ${ }^{43}$ The mechanical coupling between the solvent and molecule during the $\mathrm{B} \leftarrow \mathrm{X}$ transition is very different for these two geometries, as has been extensively investigated theoretically ${ }^{44}$ in relation to the oneatom cage effect. ${ }^{45}$ Although for the T-geometry the potential along the solvent coordinate is quite similar in the $\mathrm{X}$ and $\mathrm{B}$ states, in the linear complex the potential minimum in the $\mathrm{X}$ state is replaced by a repulsive wall in the B state. ${ }^{46}$ This difference in absorption and fluorescence spectra of the binary complex leads to sharp lines for the T-geometry and a broad continuum for the linear geometry. ${ }^{43}$ The same considerations are applicable for the liquid-phase RR spectrum. In terms of the time-circuit diagram of the $R_{3}$ correlation, the difference between the $\mathrm{T}$ - versus the linear geometry is the contraction of the $t_{2}$ interval. Thus, in the linear complex, the coherence loop consisting of a short propagation on the B state and a jump back to the $X$ state will instantaneously drive the soft $I_{2}-R g$ coordinate, which in turn is strongly coupled to the rest of the solvent modes and cannot recur. The entire circuit is then contracted to the coherence loops, which on the basis of the spectral width is limited to $25 \mathrm{fs}$. In contrast, for the T-geometry, on the basis of the line widths of overtones in the liquid phase, $t_{2} \sim 1 \mathrm{ps}$, with a weak dependence on vibrational overtones. ${ }^{17,18}$ The latter observation suggests that the line widths, and therefore the decay of the correlation function due to the population evolution on the ground electronic state, are determined by vibrational dephasing rather than relaxation.

The absence of any reported hot luminescence in the liquid phase, which should resemble the solid-state profile, is consistent with the fact that predissociation rates of the B state are an order of magnitude faster in the liquid and therefore this fluorescence intensity will be proportionally weaker.

D. Cold Luminescence, I*I* $\rightarrow$ II Transitions. The dominant features in the SLE spectra of Figures 2-4 can be assigned to the double spin-flip I*I* $\rightarrow$ II transitions. The single spin-flip transitions from the same upper states, the I*I* $\rightarrow I^{*} \mathrm{I}$ transitions, were previously reported for both solid state and liquid-phase trapping of the otherwise unbound excited state. ${ }^{24}$ These are dipole allowed transitions, with a radiative lifetime of $1-3 \mu \mathrm{s}$, comparable to the $\mathrm{B} \rightarrow \mathrm{X}$ transition. In the present, two main bands are observed in each of the matrixes, with additional weaker features in between. In the lattice, the internuclear distance in I*I* will be strictly determined by the cage, and this is consistent with the observation that the emissions undergo successive $300 \mathrm{~cm}^{-1}$ red shifts in sequence, from $\mathrm{Ar}$ to $\mathrm{Kr}$ to $\mathrm{Xe}$. At the large internuclear separations of relevance, at $R_{\mathrm{e}}=4-4.5 \AA{ }^{42}$ the optical transitions must obey strict Hund's case (c) rules, i.e., $\Delta \Omega=0, \pm 1$, along with dipolar selection $\mathrm{u} \leftrightarrow \mathrm{g}$. From the three upper states, ${ }^{1} \Sigma^{-}\left(0_{\mathrm{g}}{ }^{+}\right),{ }^{3} \Sigma^{+}\left(0_{\mathrm{u}}{ }^{-}\right)$, ${ }^{3} \Delta\left(1_{\mathrm{u}}\right)$, generated by the $\mathrm{I}\left({ }^{2} \mathrm{P}_{1 / 2}\right)+\mathrm{I}\left({ }^{2} \mathrm{P}_{1 / 2}\right)$ pair, seven transitions are possible to the $\mathrm{I}\left({ }^{2} \mathrm{P}_{3 / 2}\right)+\mathrm{I}\left({ }^{2} \mathrm{P}_{3 / 2}\right)$ limit. These are $1_{\mathrm{u}} \rightarrow \mathrm{a}\left(1_{\mathrm{g}}\right)$, $1_{\mathrm{u}} \rightarrow{ }^{3} \pi_{\mathrm{g}}\left(2_{\mathrm{g}}\right), 1_{\mathrm{u}} \rightarrow \mathrm{a}^{\prime}\left(0_{\mathrm{g}}{ }^{+}\right), 1_{\mathrm{u}} \rightarrow \mathrm{X}\left(0_{\mathrm{g}}{ }^{+}\right), 0_{\mathrm{u}}^{-} \rightarrow \mathrm{a}\left(1_{\mathrm{g}}\right), \mathrm{O}_{\mathrm{g}}{ }^{+} \rightarrow$ $\mathrm{A}\left(1_{\mathrm{u}}\right)$, and $0_{\mathrm{g}}{ }^{+} \rightarrow \mathrm{B}^{\prime \prime}\left(1_{\mathrm{u}}\right) .{ }^{47}$ As reference, in Figure 13 we give the dipole coupled potentials in an unperturbed lattice, within the diatomics-in-molecules (DIM) construct of Batista and Coker. ${ }^{42}$ Let us first note the excitation path.

The emitting states are accessed via sequential two-photon excitation with $20000 \mathrm{~cm}^{-1}$ radiation, and the intermediate state shows a lifetime of $2 \mathrm{~ms}$. Given the long delay, the re-excitation must be from a vibrationally relaxed state. Given the $5000 \mathrm{~cm}^{-1}$ 
TABLE 1: $\mathbf{I}^{*} \mathbf{I}^{*} \rightarrow \mathbf{I}_{2}$ Transition Parameters ${ }^{a}$

\begin{tabular}{|c|c|c|c|c|c|c|c|}
\hline & \multicolumn{2}{|c|}{$\mathrm{Ar}$} & \multicolumn{3}{|c|}{$\mathrm{Kr}$} & \multicolumn{2}{|c|}{$\mathrm{Xe}$} \\
\hline & $\begin{array}{c}V_{0} \\
\mathrm{~cm}^{-1} \\
\end{array}$ & $\begin{array}{c}V_{1} \\
\mathrm{~cm}^{-1} / \AA \\
\end{array}$ & $\begin{array}{c}V_{0} \\
\mathrm{~cm}^{-1} \\
\end{array}$ & $\begin{array}{c}V_{1} \\
\mathrm{~cm}^{-1} / \AA\end{array}$ & $\begin{array}{c}V_{2} \\
\mathrm{~cm}^{-1} / \AA^{2} \\
\end{array}$ & $\begin{array}{c}V_{0} \\
\mathrm{~cm}^{-1} \\
\end{array}$ & $\begin{array}{c}V_{1} \\
\mathrm{~cm}^{-1} / \AA\end{array}$ \\
\hline $1_{\mathrm{u}} \rightarrow \mathrm{a}\left(1_{\mathrm{g}}\right)$ & $15530(17180)$ & 1300 & $15325(16280)$ & 1800 & 2400 & $14980(16000)$ & 800 \\
\hline $0_{\mathrm{g}}^{+} \rightarrow \mathrm{B}^{\prime \prime}\left(1_{\mathrm{u}}\right)$ & $14640(15930)$ & 1600 & $14480(15700)$ & 1235 & & $14280(15420)$ & 1635 \\
\hline $0_{\mathrm{g}}^{+} \rightarrow \mathrm{A}\left(1_{\mathrm{u}}\right)$ & & & $14700(15790)$ & 1100 & & $14630(15650)$ & 1100 \\
\hline $0_{\mathrm{u}}^{-} \rightarrow \mathrm{a}\left(1_{\mathrm{g}}\right)$ & & & $14895(1600)$ & 1200 & & $15145(15869)$ & 2300 \\
\hline
\end{tabular}

${ }^{a}$ The experimental spectra are simulated through the one-dimensional inversion of a Gaussian upper state distribution, and a Taylor expansion of the lower state potential, eq 9 of text. The observed transition energies, $V_{0}$, are referenced to the DIM potentials of Batista and Coker for an unrelaxed lattice, which are provided in parenthesis.

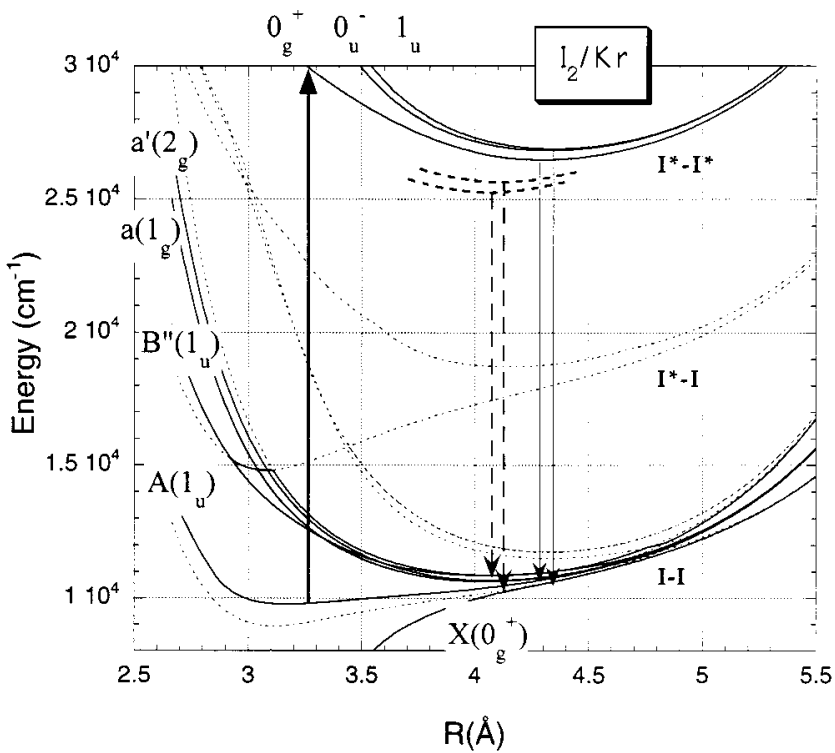

Figure 13. The dipole coupled potentials for excitation and emission over the I*I* $\rightarrow \mathrm{I}-\mathrm{I}$ transitions in $\mathrm{Kr}$. The reference potentials are from the DIM treatment of ref 42 . The emitting states are reached by twophoton excitation via the $0_{\mathrm{g}}{ }^{+} \leftarrow \mathrm{A}$ shown by the up-arrow. The experiment suggests a significant reduction of $\sim 1000 \mathrm{~cm}^{-1}$ for the transition energy in emission, which occurs after cage relaxation around the excited state (see Table 1 of text). The potentials are for a perfect lattice.

Stokes shift between excitation and emission, and because the emission terminates at the $\mathrm{I}\left({ }^{2} \mathrm{P}_{3 / 2}\right)+\mathrm{I}\left({ }^{2} \mathrm{P}_{3 / 2}\right)$ limit, the intermediate state must be below this. The $\mathrm{A}\left({ }^{3} \mathrm{P}_{1 \mathrm{u}}\right)$ and $\mathrm{A}^{\prime}\left({ }^{3} \mathrm{P}_{2 \mathrm{u}}\right)$ states are the only candidates. There are no dipole allowed transitions from $\mathrm{A}^{\prime}$ to the $\mathrm{I}^{*} \mathrm{I}^{*}$ limit, therefore we are left with $\mathrm{A}$ as the only candidate. The $\mathrm{A}$ and $\mathrm{A}^{\prime}$ states have been well-studied in the past. ${ }^{25}$ It is known that the A state relaxes radiatively with $\tau=100 \mu \mathrm{s}-200 \mu \mathrm{s}$ in $\mathrm{Kr}$; while the $\mathrm{A}^{\prime}$ state shows a biexponential decay with time constants ranging between 2 and $7 \mathrm{~ms}$ and 40-70 ms depending on matrix and concentration. ${ }^{25,48,49}$ At least one of these time constants represents the filling of the $\mathrm{A}^{\prime}$ state, from a reservoir suspected to be the $\mathrm{O}_{\mathrm{u}}{ }^{-}$ state. The observed $2 \mathrm{~ms}$ lifetime of the intermediate state in our measurement agrees with the faster of the $\mathrm{A}^{\prime}$ time constants, although both energetically and on the basis of selection rules the intermediate state must be identified as A. In effect, the population that leaks into A must also pass through $\mathrm{A}^{\prime}$, even though this would be difficult to observe in the $A^{\prime} \rightarrow X$ emission. This has already been suggestion by Böhling et al., who argue that $\mathrm{A}$ and $\mathrm{A}^{\prime}$ remain strongly coupled until the very bottom of the $A^{\prime}$ state, where they decouple due to the poor Franck-Condon factors. As indicated in Figure 13, the I*I*$\left(0_{\mathrm{g}}{ }^{+}\right) \leftarrow \mathrm{A}\left(1_{\mathrm{u}}\right)$ transition is coincident with the $\mathrm{Ar}^{+}$-ion laser lines used, near $20000 \mathrm{~cm}^{-1}$.

The excited state is prepared with an excess energy of $\sim 3000$

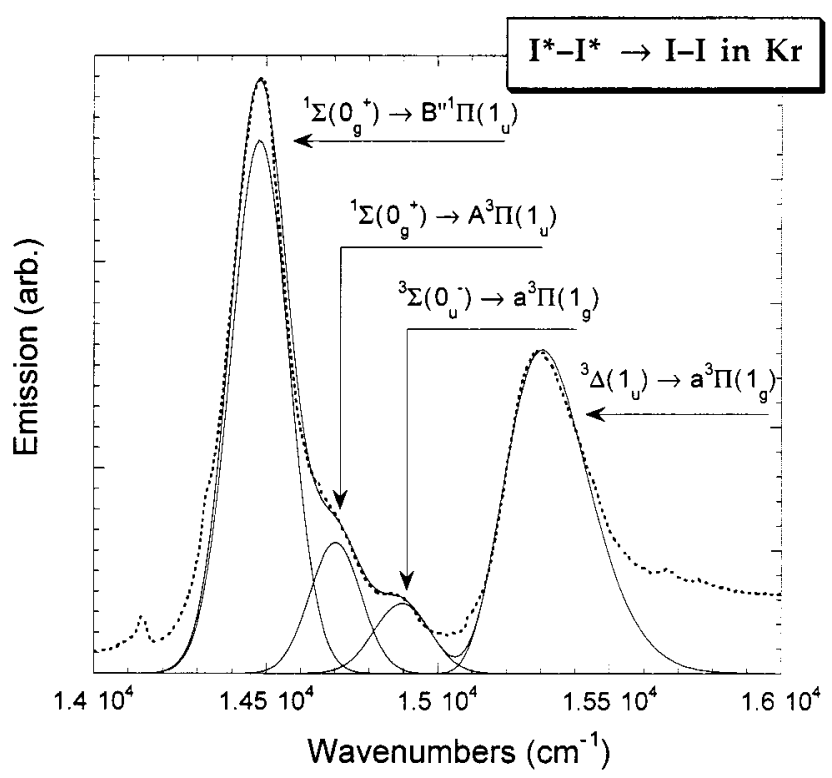

Figure 14. Assignment and simulation of the $I^{*} I^{*} \rightarrow I-I$ cold luminescence in $\mathrm{Kr}$. The line shapes are from the one-dimensional reflection using parameters provided in Table 1 of text.

$\mathrm{cm}^{-1}$, sufficient to populate all three I*I* states during vibrational relaxation. Indeed, a close inspection of the emission spectra in $\mathrm{Kr}$, which beside the two main bands clearly shows shoulders, suggests that all three upper states are populated and emit. The blue-most of these transitions would be $1_{\mathrm{u}} \rightarrow \mathrm{X}\left(0_{\mathrm{g}}{ }^{+}\right)$, followed by $1_{\mathrm{u}} \rightarrow \mathrm{a}\left(1_{\mathrm{g}}\right), \mathrm{a}^{\prime}\left(2_{\mathrm{g}}\right)$, and $0_{\mathrm{g}}{ }^{+}$with three terminal states that are expected to be coalesced in this limit. The $\Delta \Omega=0$ propensity for transitions would argue that the $1_{\mathrm{u}} \rightarrow 1_{\mathrm{g}}$ transition is the strongest. The red-most transition from $I^{*} I^{*}$ would be expected to be $0_{\mathrm{g}}{ }^{+} \rightarrow \mathrm{B}^{\prime \prime}\left(1_{\mathrm{u}}\right)$, followed by $\mathrm{O}_{\mathrm{g}}{ }^{+} \rightarrow \mathrm{A}\left(1_{\mathrm{u}}\right)$. With this assignment, it is possible to reconstruct the spectral profile in $\mathrm{Kr}$, using the previously recommended parameters of the exponential-reflected-Morse construct for $\mathrm{I}^{*} \mathrm{I}^{*}$ (harmonic parameters $\omega_{\mathrm{e}}=58 \mathrm{~cm}^{-1}, r_{\mathrm{e}}{ }^{\prime}=4.26 \AA$ ). ${ }^{24}$ Within the $\sim 0.2 \AA$ span of the Gaussian upper state internuclear separation probability, $P(q)=\exp \left(-\pi \mu \omega_{\mathrm{e}} c q^{2} / \hbar\right)$, a Taylor expansion of the lower potential at the upper state minimum:

$$
E=V_{0}+V_{n} q+V_{2} q^{2}
$$

where $q=r-r_{\mathrm{e}}{ }^{\prime}$, is sufficient to reproduce the observed band shapes (see Figure 14). In essence, what is extracted from such a fit is the potential difference between upper and lower state, $V_{0}$, and the slope, $V_{1}$, and curvature, $V_{2}$, of the lower state at the excited-state minimum. These parameters are collected in Table 1 , including the $\mathrm{V}_{0}$ values of the reference potentials. Note that the linear term of the power expansion is sufficient for all but the $1_{\mathrm{u}} \rightarrow 1_{\mathrm{g}}$ transition, for which case the skewing is reproduced by the inclusion of the quadratic term $V_{2}$. This 


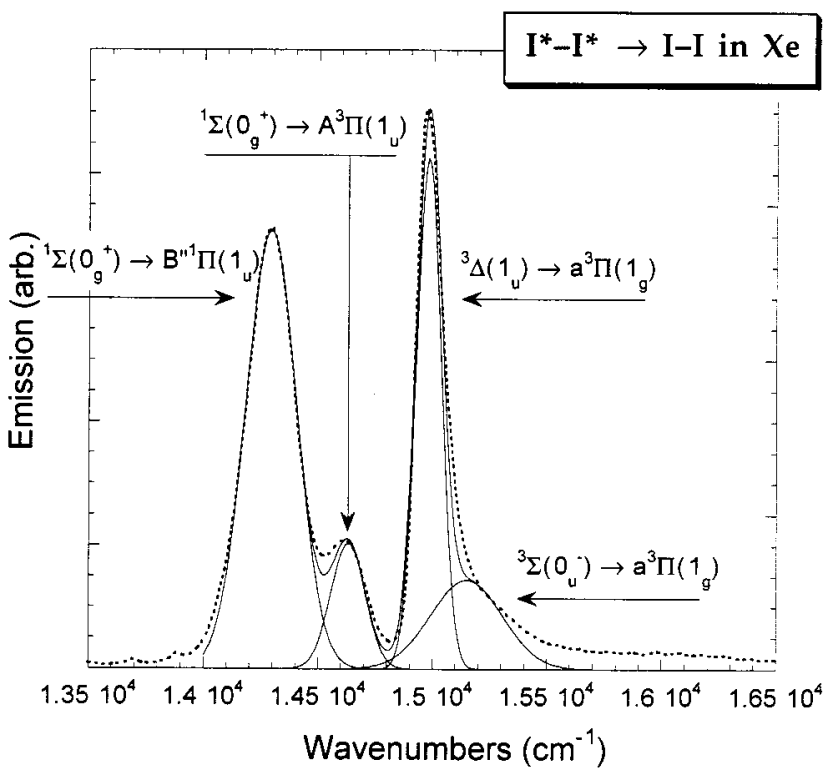

Figure 15. Assignment and simulation of the I*I* $\rightarrow$ I-I cold luminescence in Xe.

may simply be the result of the congestion in the lower states, because the transition must have contributions from $1_{\mathrm{u}} \rightarrow 2_{\mathrm{g}}$ and $1_{\mathrm{u}} \rightarrow 0_{\mathrm{g}}$ as well. The same ordering of the transitions, except for the $0_{\mathrm{u}}{ }^{-}$emission, and similar parameters reproduce the spectrum in Xe see Figure 15. Note that the experimental band positions, $V_{0}$ values in Table 1 , are consistently red-shifted by $\sim 1000 \mathrm{~cm}^{-1}$ relative to theory. This discrepancy can in part be ascribed to the fact that the emission occurs after lattice relaxation around the excited state, whereby the upper states will be shifted to lower energy, as indicated in Figure 13, and the lower potentials may be further repelled. However, the magnitude of the discrepancy is large, and may indicate a stronger guest host interaction than predicted by the DIM construct, such as may be expected from partial charge-transfer character in the upper states.

\section{Conclusions}

The SLE spectra of matrix-isolated iodine provide a useful model to illustrate differences between coherent Raman, hot luminescence, and vibrationally relaxed cold luminescence. We discussed these differences from the perspective of the semiclassical molecular dynamics, which allows a direct connection between spectral observables and atomistic mechanics. The analysis of the hot luminescence through classical trajectories is justified, and we learn from this analysis that the B state of iodine predissociates on the time scale of $3-5 \mathrm{ps}$, and that although created near its dissociation limit the molecular does not start forming eigenstates until it reaches $\mathrm{v}=6$, and even then the states are subject to strong vibrational dephasing. The spectral signature of the latter is the formation of structure in the emission spectrum, although predissociation preempts the formation of a proper progression. The analysis of the solidstate hot luminescence, and the extraction of time scales associated with the spectral features, allows a contrast with the liquid phase spectra, and we conclude that they are strictly due to RR. The presence of discrete and continuous RR spectra must then be ascribed to complexation in the liquid phase, the linear complexes being associated with the continuous spectrum. Explicit simulations of this spectroscopy, through implementation of the semiclassical time circuits, is now in progress.

The dominant features in the continuous wave excited emission spectra of matrix-isolated iodine are due to the $I^{*}-I^{*}$ $\rightarrow$ I-I transitions, which are reached via sequential two-photon excitation, with the intermediate state identified as $\mathrm{A}\left({ }^{3} \mathrm{P}_{1 \mathrm{u}}\right)$. The transitions are assigned on the basis of selection rules and expected ordering of the states in the asymptotic region. These frequency domain data are quite informative regarding the nonadiabatic couplings among the nested potentials of iodine. Although only the $0_{\mathrm{g}}{ }^{+}$excited state is accessible optically, all three excited states are implicated in the emission. This would imply that the excess kinetic energy with which the upper state is formed is sufficient for the interconversion; however, once vibrationally relaxed, these states do not communicate on the radiative time scale of $3 \mu \mathrm{s}$. For the lower spin state manifold, although the B state predissociates in $3-5 \mathrm{ps}$, there are reservoir states that do not interconvert for $2 \mathrm{~ms}$, despite the density of the manifold. The spectroscopic data provided here are quite useful for the interpretation of time-resolved studies of nonadiabtic dynamics in this system.

Acknowledgment. This research has been made possible through grants from the NSF (CHE-9708382) and the US AFOSR (F49620-95-1-0213). The semiclassical framework, and the time-circuit diagrams offered here, are a direct off-shoot of the pioneering work carried out by Dr. M. Ovchinnikov at Irvine. We would also like to thank D. Coker and V. Batista for the DIM potentials that were used as reference in this work.

\section{References and Notes}

(1) Chuang, T. J.; Hoffman, G. W.; Eisenthal, K. B. Chem. Phys. Lett. 1974, 25, 201.

(2) Harris, A. L., Brown, J. K.; Harris, C. B. Annu. Rev. Phys. Chem. 1988, 39, 341.

(3) Scherer, N. F.; Jonas, D. M.; Fleming, G. R. J. Chem. Phys. 1993, $99,153$.

(4) Zadoyan, R.; Sterling, M.; Ovchinnikov, M.; Apkarian, V. A. J. Chem. Phys. 1997, 107, 8446.

(5) (a) Liu, Q.; Wang, J.-K.; Zewail, A. H. Nature 1993, 364, 427. (b) Wang, J. K.; Liu, Q.; Zewail, A. H. J. Phys. Chem. 1995, 99, 11309. (c) Liu, Q.; Wang, J.-K.; Zewail, A. H. J. Phys. Chem. 1995, 99, 11321.

(6) (a) Philippoz, J. M.; van den Bergh, H.; Monot, R. J. Phys. Chem. 1987, 91, 2545. (b) Philippoz, J. M.; Monot, R.; van den Bergh, H. J. Chem. Phys. 1990, 93, 8676.

(7) (a) Schroeder, J.; Troe, J. Annu. Rev. Phys. Chem. 1987, 38, 163. (b) Lienau, Ch.; Williamson, J. C.; Zewail, A. H. Chem. Phys. Lett. 1993 213, 289. (c) Lienau, Ch.; Zewail, A. H. Chem. Phys. Lett. 1994, 222, 224. (d) Lienau, Ch.; Zewail, A. H. J. Chim. Phys. 1995, 92, 566.

(8) Zadoyan, R.; Sterling, M.; Apkarian, V. A. J. Chem. Soc., Faraday Trans. 1996, 92, 1821.

(9) Zadoyan, R.; Almy, J.; Apkarian, V. A. J. Faraday Discuss. 1997, 108, 255.

(10) Bardeen, C. J.; Che, J.; Wilson, K. R.; Yakovlev, V. V.; Apkarian, V. A.; Martens, C. C.; Zadoyan, R.; Kohler, B.; Messina, M. J. Chem. Phys. 1997, 106, 8486.

(11) Howard, W. F.; Andrews, L. J. Raman Spectrosc. 1974, 2, 442.

(12) Grzybowski, J.; Anrews, L. J. Raman Spectrosc. 1975, 4, 99.

(13) Andrews, L. Appl. Spectrosc. Rev. 1976, 11, 125.

(14) Sension, R. J.; Strauss, H. L. J. Chem. Phys. 1986, 85, 3791.

(15) (a) Sension, R. J.; Kobayashi, T.; Strauss, H. L. J. Chem. Phys. 1987, 87, 6221. (b) Ibid. 1987, 87, 6233

(16) Sension, R. J.; Strauss, H. L. J. Chem. Phys. 1988, 88, 2289

(17) Xu, J.; Schwentner, N.; Chergui, M. J. Chem. Phys. 1994, 101, 7381

(18) Xu, J.; Schwentner, N.; Hennig, S.; Chergui, M. J. Raman Spectrosc. 1997, 28, 433; J. Chim. Phys. (Paris) 1995, 92, 541.

(19) Kono, H.; Lin, S. H. J. Chem. Phys. 1986, 84, 1071.

(20) (a) Jungwirth, P.; Gerber, R. B. J. Chem. Phys. 1995, 102, 8855 (b) Jungwirth, P.; Fredj, E.; Gerber, R. B. J. Chem. Phys. 1996, 104, 9332.

(21) Ovchinnikov, M.; Apkarian, V. A. J. Chem. Phys. 1996, 105, 10312.

(22) Ovchinnikov, M.; Apkarian, V. A. J. Chem. Phys. 1997, 106, 5775.

(23) Mukamel, S. Principles of Nonlinear Optical Spectroscopy; Oxford University Press: New York, 1995.

(24) Benderskii, A.; Zadoyan, R.; Apkarian, V. A. J. Chem. Phys. 1997, 107,8437

(25) (a) Macler, M.; Heaven, M. Chem. Phys. 1991, 219, 151. (b) Macler, M.; Nicolai, J.-P.; Heaven, M. C. J. Chem. Phys. 1989, 91, 674. 
(26) Tang, J.; Albrecht, A. C. Raman Spectroscopy; Szymanski, H. A., Ed.; Plenum: New York, 1970; Vol. I

(27) Huber, D. L. Phys. Rev. 1967, 158, 843; 1968, 170, 418; 1969, 178, 93; Phys. Rev. B 1970, 1, 3409.

(28) Hizhnyakov, V.; Tehver, I. Phys. Status Solidi 1967, 21, 755.

(29) Lee, S. Y.; Heller, E. J. J. Chem. Phys. 1979, 71, 4777.

(30) Mukamel, S. J. Chem. Phys. 1985, 82, 5398; Adv. Chem. Phys. 1988, $70,165$.

(31) Stock, G.; Domcke, W. J. Chem. Phys. 1990, 93, 5496.

(32) Ovchinnikov, M.; Apkarian, V. A. J. Chem. Phys. 1998, 108, 2277.

(33) Gordon, R. G. Adv. Magn. Reson. 1968, 3, 1.

(34) (a) Herman, M. F.; Kluk, E. Chem. Phys. 1984, 91, 27. (b) Kluk, E.; Herman, M. F.; Davis, H. L. J. Chem. Phys. 1984, 84, 333.

(35) (a) Sun, X.; Miller, W. H. J. Chem. Phys. 1999, 110, 6635. (b) Ibid. 1999, 111, 1788 .

(36) For an extensive discussion, see: Giulini, D.; Joos, E.; Kiefer, C.; Kupshc, J.; Stamatescu, I. O.; Zeh, H. D. Decoherence and the Appearance of a Classical World in Quantum Theory; Springer-Verlag: Berlin, 1996.

(37) Miller, W. H. Adv. Chem. Phys. 1974, 25, 69.

(38) Sun, X.; Miller, WH. J. Chem. Phys. 1997, 106, 916.

(39) Herman, M. F.; Coker, D. F. J. Chem. Phys. 1999, 111, 1801.

(40) Tellinghuisen, J. J. Chem. Phys. 1997, 106, 1305.

(41) Lamrini, M.; Cerny, D.; Churassy, S.; Crozel, P.; Ross, A. J. J. Chem. Phys. 1994, 100, 8780.
(42) Batista, V. S.; Coker, D. F. J. Chem. Phys. 1997, 106, 6923.

(43) (a) Burke, M. L.; Klemperer, W. J. Chem. Phys. 1993, 98, 1797. (b) Cockett, M. C. R.; Beattie, D. A.; Donovan, R. J.; Lawley, K. P. Chem Phys. Lett. 1996, 259, 554. (c) Burroughs, A.; Van Marter, T.; Heaven, M. C. J. Chem. Phys. 1999, 111, 2478-2483.

(44) (a) Noorbatcha, I.; Raff, L. M.; Thomson, D. L.; J. Chem. Phys. 1984, 81, 5658. (b) Beswick, J. A.; Monot, R.; Philipoz, J. M.; van den Bergh, H. J. Chem. Phys. 1987, 86, 3965. (c) de Miranda, M. P.; Beswick, J. A.; Halberstadt, N. Chem. Phys. 1994, 187, 185. (d) Fang, J. Y.; Martens, C. C. J. Chem. Phys. 1996, 105, 9072. (e) Zamith, S.; Meier, C.; Halberstadt, N.; Beswick, J. A. J. Chem. Phys. 1999, 110, 960.

(45) (a) Saenger, K. L.; McClelland, G. M.; Herschbach, D. R.; J. Phys. Chem. 1981, 85, 3333. (b) Valentini, J. J.; Cross, J. B. J. Chem. Phys. 1982 77, 572. (c) Philippoz, J. M.; van de Bergh, H.; Monot, R. J. Phys. Chem 1987, 91, 1, 2545.

(46) (a) Naumkin, F. Y.; McCourt, F. R. W. Chem. Phys. Lett. 1998, 294, 71. (b) Kunz, C. F.; Burghardt, I.; Hess, B. A. J. Chem. Phys. 1998, 109, 359.

(47) Mulliken, R. S. J. Chem. Phys. 1971, 55, 288.

(48) Beeken, P. B.; Hanson, E. A.; Flynn, G. W. J. Chem. Phys. 1983, 78,5892 .

(49) Böhling, R.; Langen, J.; Schurath, U. Chem. Phys. 1989, 130, 419 